\title{
Efficient polymer-mediated delivery of ribonucleoprotein payloads through combinatorial design \& parallelized experimentation.
}

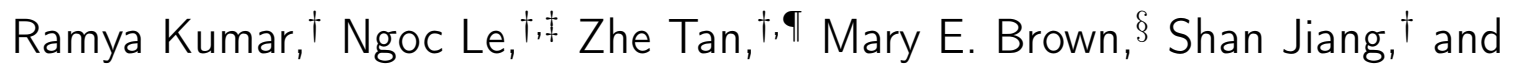 \\ Theresa M. Reineke ${ }^{* \dagger}$ \\ $\dagger$ Department of Chemistry, University of Minnesota Twin Cities \\ $\ddagger$ Present address: Bayramoglu Law Offices, 1540 W.Warm Springs Road, Henderson, NV \\ \Present address: Modern Meadow Inc., 111 Ideation Way, Nutley, NJ \\ $\S$ University Imaging Centers, University of Minnesota Twin Cities \\ E-mail: treineke@umn.edu
}

\begin{abstract}
Genome editing is almost completely reliant on viral delivery to achieve therapeutic goals, hindering widespread clinical adoption. Chemically defined delivery vehicles such as cationic polymers are versatile alternatives to engineered viruses, but their clinical translation hinges on rapidly exploring vast chemical design spaces and deriving structure-function relationships governing delivery performance. Here, we discovered a polymer for efficient ribonucleoprotein (RNP) delivery through combinatorial polymer design and parallelized experimental workflows. A chemically diverse library of 43 statistical copolymers was synthesized via combinatorial RAFT polymerization, realizing systematic variations in physicochemical properties. We
\end{abstract}


selected cationic monomers that varied in their $p K_{a}$ values (8.1-9.2) as well as in the steric bulk and lipophilicity of their alkyl substituents. We also incorporated co-monomers of varying hydrophilicity and elucidated the roles of protonation equilibria and hydrophobic-hydrophilic balance. We screened our multiparametric vector library through image cytometry and rapidly uncovered a hit polymer (P38), which outperforms state-of-the-art commercial transfection reagents, achieving nearly $60 \%$ editing efficiency via non-homologous end-joining. Structure-function correlations underlying editing efficiency, cellular toxicity, and RNP uptake were probed through unbiased statistical learning approaches to uncover the physicochemical basis of P38's performance. Although cellular toxicity and RNP uptake were solely determined by polyplex size distribution and protonation degree respectively, these two polyplex design parameters were found to be inconsequential during RNP delivery. Instead, polymer hydrophobicity and the Hill coefficient, a parameter describing cooperativity-enhanced polymer deprotonation, were identified as the critical determinants of RNP delivery. Our unconventional approach not only discovered a novel polymeric vehicle that may have remained inaccessible to chemical intuition, but also yielded statistically derived design rules to guide the synthesis of future polymer libraries.

\section{Keywords}

genome editing, polymeric gene delivery, CRISPR, RNP delivery, non-viral vectors, high-throughput experimentation, combinatorial design, statistical learning, structure-activity relationships, random forest classifiers. 
Genome editing platforms based on clustered regularly interspersed palindromic repeats (CRISPR) have transformed the therapeutic landscape for diseases where healthy cellular states can be restored through the deletion, insertion, or repair of genetic sequences. ${ }^{1,2}$ Recent clinical trials of investigational gene therapeutics for $\beta$-thalassemia and sickle cell disease suggest that the CRISPR gene editing platform is safe and efficacious. ${ }^{3,4}$ Additional clinical trials are underway to develop CRISPR-focused therapeutics for debilitating conditions such as Duchenne's muscular dystrophy (DMD), Leber congenital amaurosis (LCA), and chimeric antigen receptor T-cell (CAR-T) therapies for cancer. ${ }^{5,6}$ Despite the immense curative potential of CRISPR, widespread clinical deployment faces an uncertain outlook due to excessive reliance on engineered viral vectors, which are the platforms of choice for delivering messenger RNA (mRNA), plasmid DNA (pDNA), and small interfering RNA (siRNA). ${ }^{7,8}$ Production of clinical grade viruses involves prohibitive costs and logistical bottlenecks while the scale-up of viral vectors for large patient populations has to contend with regulatory issues. In addition to manufacturing and regulatory delays caused by viral vectors, they are limited in their cargo capacity, and this size ceiling is particularly problematic in the context of bulky cargoes. ${ }^{9}$ For example, adeno-associated viruses, a widely used therapeutic vehicle that is considered to be among safest viral vectors, can only accommodate $4.5 \mathrm{kbp}$, limiting its adoption for multi-component CRISPR cargoes. $^{10}$ Although advances in viral vector engineering have minimized occurrences of carcinogenic mutations, genomic integration, and fatal systemic inflammatory responses, these risks are amplified when repeated dosing or large dosages are involved. ${ }^{11}$ In order to engineer safe, scalable and affordable CRISPR therapeutics, we must meet the urgent need for replacing viral gene carriers with synthetic materials. ${ }^{12}$

Developing biomaterials to deliver gene therapeutics is a design challenge spanning several length scales, temporal horizons, and biological barriers within a delicate delivery cascade. ${ }^{13}$ As a first step, polymeric vehicles are expected to condense the CRISPR payloads (mRNA, pDNA or ribonucleoproteins) into discrete nanosized polyelectrolyte 
complexes termed polyplexes. Polyplexes must subsequently navigate extracellular barriers such as serum DNAases (or RNAases) and reticuloendothelial system (RES) clearance, as well as intracellular barriers such as endosomal interrogation and lysosomal degradation. ${ }^{14}$ Finally the cargo must be released within a narrow spatiotemporal window that is optimal for payload translocation to the nucleus, where they can undergo further processing and realize targeted edits. ${ }^{15}$ In addition to fulfilling exacting specifications for safety, efficiency and cost-effectiveness, synthetic vectors must minimize immune activation and cellular toxicity. ${ }^{16}$ Since non-viral gene delivery imposes stringent design specifications and demands precise control over interfacial properties, polymer chemistry is uniquely suited to generate vehicles for nucleic acid cargoes. Advances in controlled radical polymerization methods such as reversible addition fragmentation-transfer polymerization (RAFT) have enabled hitherto unprecedented access to diverse polymer architectures. A continually expanding monomer scope and progress in fine-tuning polymerization kinetics have together ensured that we can synthesize polymers of any desired chemical composition, architecture, and molecular weight distributions, allowing us to create multi-functional gene delivery vehicles with exquisitely tailored properties. ${ }^{17}$ Unlike engineered viruses, the mass-manufacture of polymers does not present logistical and economic challenges.

By incorporating monomers in different ratios, architectures, and lengths, we can generate almost infinite structurally unique design possibilities. Although polymer chemists can produce portfolios of macromolecules on demand, it is difficult to predict from first principles whether the synthesized polymers will satisfy design requirements for a given therapeutic application. Since the biological milieu in which payloads are delivered by polymeric vectors is complex, ab initio prediction of gene editing efficiency directly from polymer structure is almost impossible. For example, the proton-sponge hypothesis ${ }^{18}$ has confounded efforts to acquire a nuanced physical understanding of the precise role played by polycations in intracellular trafficking of their payloads. ${ }^{19}$ Similarly, studies exploring the role of design facets such as polymer hydrophobicity, ${ }^{20-22}$ PEGylation, ${ }^{23}$ protein corona 
formation, ${ }^{24}$ and polyplex diameter ${ }^{25}$ during transfection have seldom converged on a single answer. With neither theoretical models not heuristic knowledge providing reliable experimental guidance, it is challenging to explore the polymer design space in a manner that minimizes experimental effort and accelerates polymer discovery. Recognizing the urgent clinical need for non-viral vectors, we deployed parallelized experimentation and statistical learning in tandem to discover high-performing polymeric vehicles rapidly. We draw attention to previous studies where combinatorial libraries of cationic lipids, ${ }^{26-31}$ polymers, ${ }^{23,32-40}$ and gold nanoparticles, ${ }^{41-43}$ were screened to identify high-performing synthetic gene delivery vectors rapidly. Although the application of high-throughput approaches is becoming more widespread in gene delivery, ${ }^{44}$ the elucidation of structure-function relationships from these large datasets and the development of predictive models that identify correlations between biological responses and polymer attributes has received scant attention. In contrast to previous studies that focused mainly on exploiting serendipity to discover high-performing materials, our study goes beyond hit identification by answering the following questions: (1) Which interfacial properties favor efficient intracellular delivery? (2) How do we design polymers in order to realize these optimal properties?

We integrated combinatorial polymer design, parallel synthesis, image cytometry, high-throughput biological assays, and statistical learning into a streamlined polymer discovery workflow (Figure 1). Through this approach, we rapidly explored a rich multidimensional chemical design space and identified a polymer for highly efficient intracellular ribonucleoprotein (RNP) delivery. Four cationic monomers with distinct charge centers and $p K_{a}$ values were combined with three co-monomers of varying hydrophilicity to generate a library of 43 statistical copolymers via combinatorial RAFT polymerization. We probed whether differences in amine basicity and alkyl substituent lengths would alter polycation protonation equilibria and ultimately influence payload binding, cellular internalization, and intracellular payload release. In addition to variations 


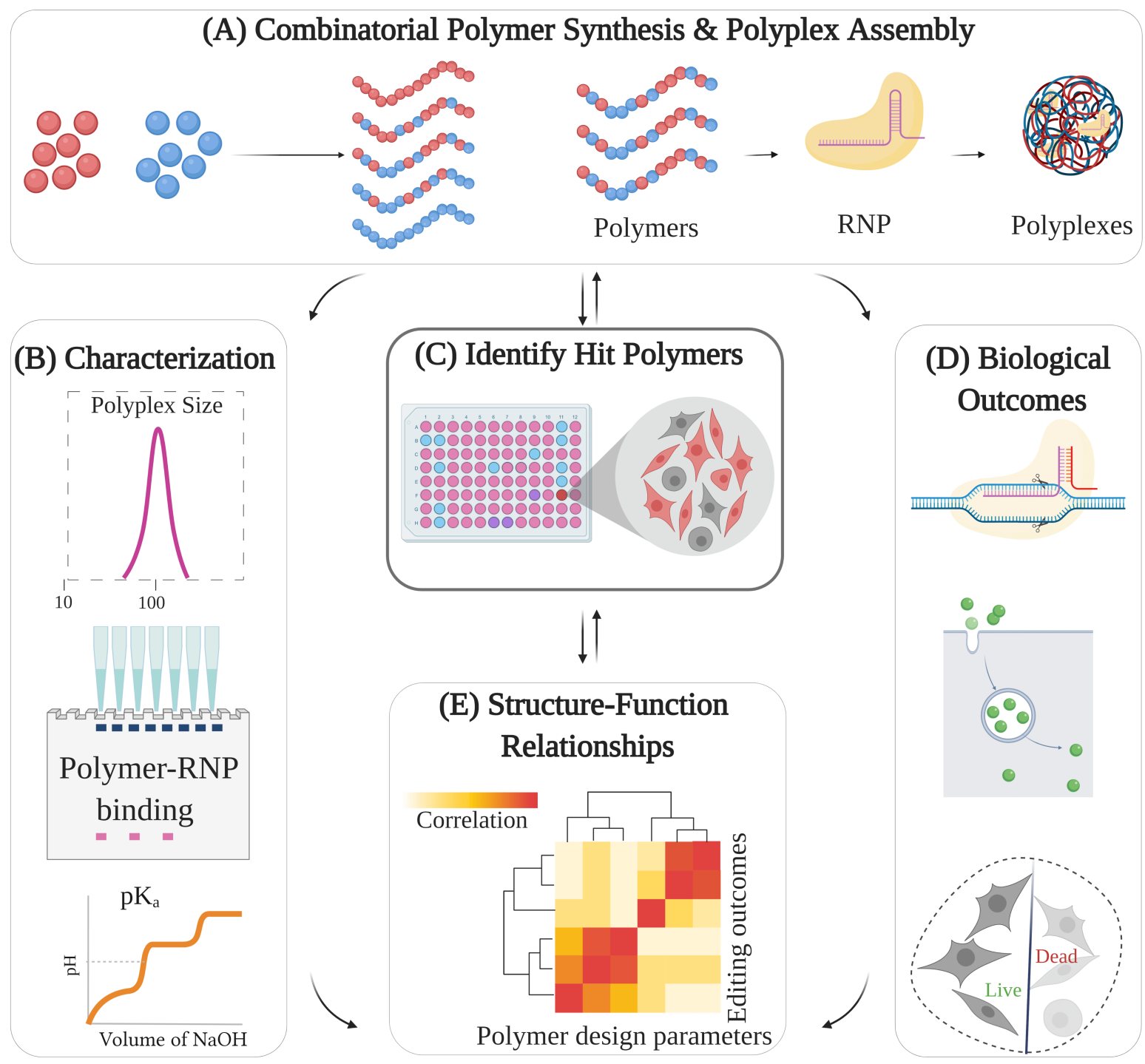

Figure 1: Overview of workflow used in this contribution. (A) Following combinatorial RAFT polymerization using a parallel synthesizer, polymers were assembled with RNP payloads. (C) Gene editing efficiency was screened using high-throughput biological assays. (B) Polymers were characterized in parallel to probe protonation behavior, RNP binding affinity, polyplex size distribution, and charge density. (D) Library-wide measurements of polyplex internalization, and toxicity were completed in parallel. (E) Finally, statistical learning tools were deployed to mine experimental datasets and generate structure-function maps correlating polymer attributes with RNP delivery, cellular toxicity, and RNP uptake. 
in basicity stemming from the type of charge center employed (primary or tertiary amines), we expected hydrophobic interactions to prove significant, since the alkyl substituents of the tertiary amine methacrylates vary in steric bulk and lipophilicity. ${ }^{20,45}$ Further, we evaluated the impact of co-monomer hydrophilicity and the degree of co-monomer incorporation on polyplex size distributions, payload binding equilibria, cellular uptake of polyplexes, toxicity, and intracellular delivery. We identified a lead structure (P38), which was subsequently benchmarked against four state-of-the-art commercial transfection reagents. Sanger sequencing confirmed that P38 outperformed every commercial reagent, achieving 58\% indel formation, an editing efficiency that is two-fold higher than Lipofectamine CRISPRMAX and JetCRISPR. Having established that P38 is a promising delivery vehicle for ex vivo gene editing, we employed statistical learning tools to identify structural drivers associated with cellular toxicity, editing efficiency, and cellular internalization of RNP payloads. Through tree-based classification schemes, we found that the structural basis for cellular internalization and payload delivery diverged considerably. While polycation protonation favored polymer-mediated RNP uptake, it did not contribute to efficient intracellular delivery of RNPs. Instead, we must engineer hydrophobic polymers that deprotonate with a high degree of cooperativity to ensure that RNP payloads are rapidly unpackaged within the cytosol subsequent to uptake. To implement these model-derived design rules in future polymer libraries, we must: (1) boost polymer hydrophobicity by selecting co-monomers possessing moderately hydrophilic motifs (2) incorporate bulky alkyl groups within tertiary amine methacrylates to promote hydrophobic collapse and cooperative polymer deprotonation. Our workflow: (1) speedily discovers "hit polymers" from unexpected regions in chemical space, allowing us to realize superlative editing performance through chemically well-defined non-viral delivery modalities, (2) learns from serendipitous discoveries, identifies design rules latent within hit polymers, and generates overarching structure-function correlations that can be broadly applied to design future polymer libraries. Our powerful discovery pipeline can be applied 
across diverse cell types and nucleic acid cargoes to individually tailor the properties of polymeric vehicles for varied clinical goals, transforming the therapeutic landscape for genome editing.

\section{Results \& Discussion}

Parallel synthesis and characterization of a combinatorially designed polymer library

(A)

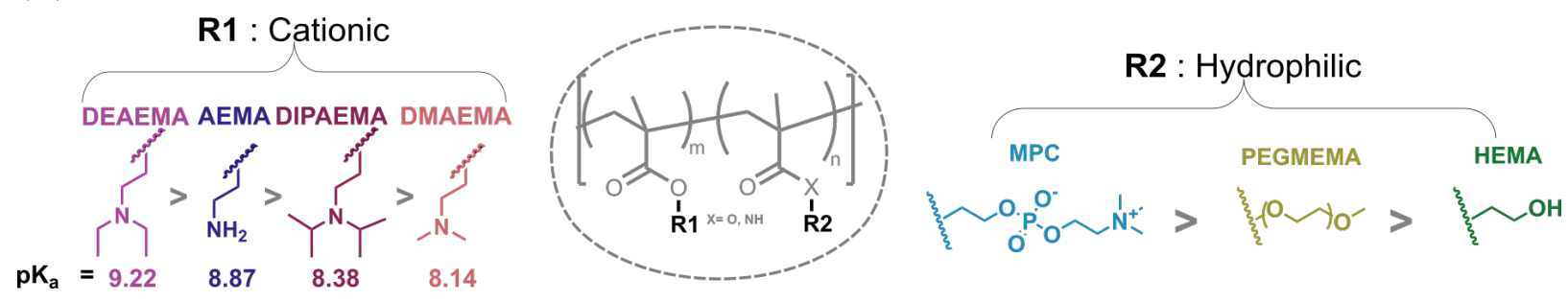

(B)
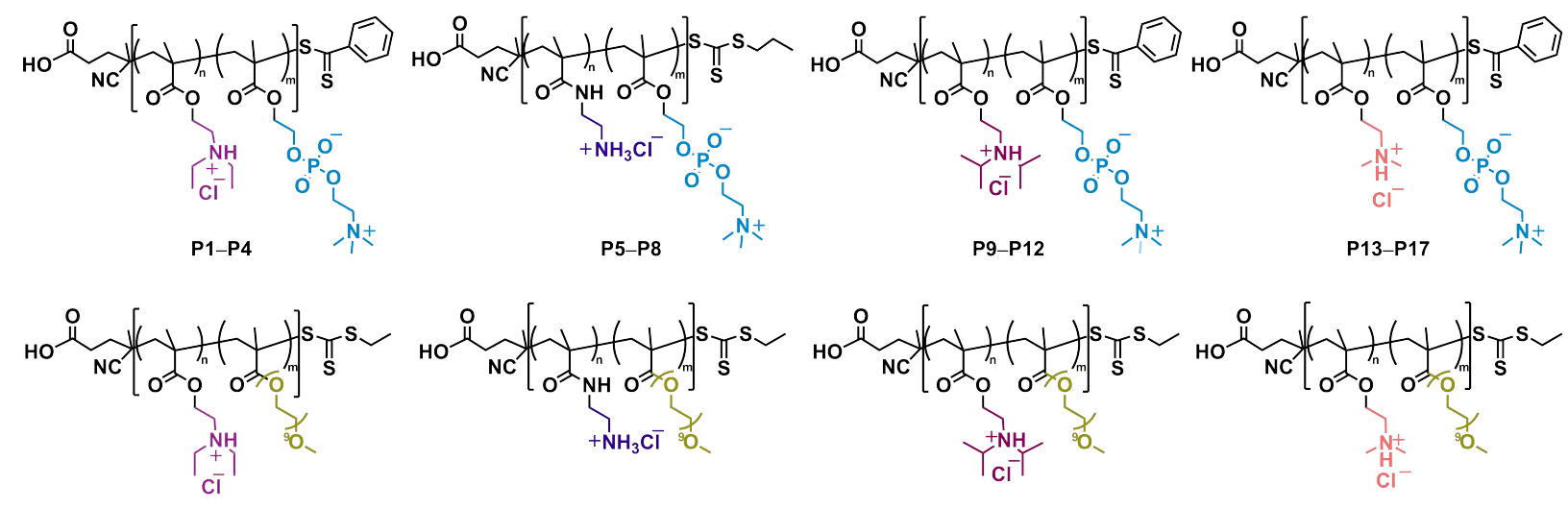

P18-P20

P21-P23

P24-P26

P27-P30

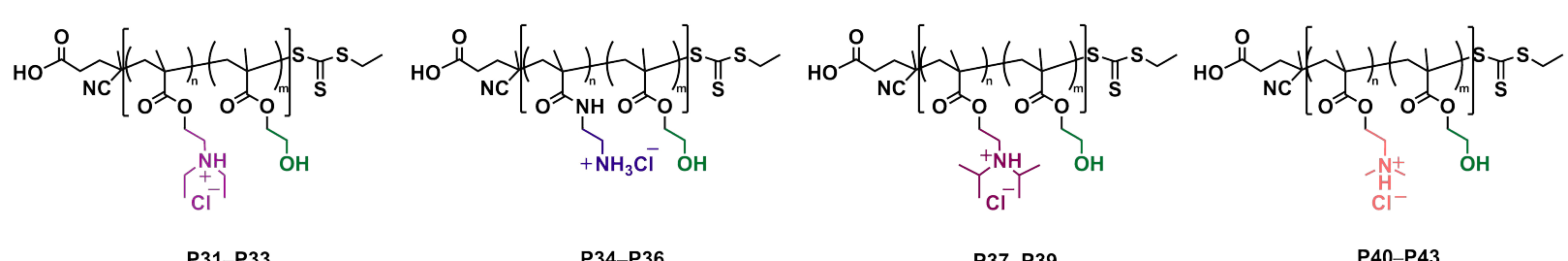

Figure 2: Scheme for combinatorial RAFT polymerization. Cationic monomers $\left(p K_{a}\right.$ values between 8.14 to 9.22 ) were copolymerized with neutral monomers of varying hydrophilicity. The targeted cationic monomer incorporation was varied systematically from $0 \%$ to $100 \%$ in $25 \%$ increments, ultimately giving rise to 43 statistical copolymers of systematically varied interfacial properties. 
While cationic monomers confer positive charge densities that mediate nucleic acid condensation, their reaction partners may alleviate toxicity, prolong polyplex colloidal stability, or modulate the binding equilibrium of the polyplexes. Pairing three hydrophilic monomers with four cationic monomers gives rise to twelve binary copolymers. Within each binary copolymer, we varied the incorporation of cationic monomer to target cationic monomer incorporations of $100 \%, 75 \%, 50 \%, 25 \%$, and $0 \%$ (Figure 2). Overall, a library of 43 well-defined copolymers was generated using reversible addition fragmentation chain transfer (RAFT) polymerization (Table 1). Subsequent to synthesis, we measured (1) polymer composition (0\%-100\% cationic), (2) molecular weight distribution (9-49 kDa), (3) $\zeta$-potential (-15 mV to $+23 \mathrm{mV}$ ), and (4) $p K_{a}(5.9-9)$ of all polymers within the library. Systematic variations to polymer composition were introduced through combinatorial design, resulting in polyplex hydrodynamic radii ranging from $6 \mathrm{~nm}$ to nearly $2 \mu \mathrm{m}$ (Figures S12 to S14), and RNP binding affinities spanning strong, weak and intermediate degrees of complexation (Figures S15 to S17).

In most cases, the composition of polymerized products recapitulated that of the monomer feed, simplifying the realization of targeted cationic incorporation. Despite reaching high conversion (80\%-90\%), we were able to achieve excellent control over the controlled radical polymerization kinetics $(\mathrm{D}<1.2$ ) for almost the entire library while also obtaining the desired degree of polymerization (a minimum $M_{n}$ of $10 \mathrm{kDa}$ was targeted and was attained in most instances). We also performed $\zeta$-potential measurements and $p K_{a}$ titrations (Table 1) to quantify surface potential and protonation equilibria, respectively. The cationic monomers vary in the type of charge center (primary vs tertiary amines) and encompass a range of $p K_{a}$ values from 8-9 (Figure 2); however the $p K_{a}$ values of the resultant (co)polymers spanned a much broader range between 5.9-9 (Table 1). While cationic homopolymers typically exhibited high charge densities (as measured by the $\zeta$-potential), the installation of extremely hydrophilic functionalities such as PEG or MPC typically resulted in sharp declines in surface potentials. 
Table 1: Characterization via $\mathrm{NMR}^{\mathrm{a}}, \mathrm{SEC}^{\mathrm{b}}$, titration $^{\mathrm{c}}$ and electrophoresis ${ }^{\mathrm{d}}$

\begin{tabular}{|c|c|c|c|c|c|c|c|c|c|}
\hline Entry & Polymer & $m$ & $n$ & $\begin{array}{l}\% \text { cat. } \\
\text { (target) }\end{array}$ & $\%$ cat $^{\mathrm{a}}$ & $\begin{array}{l}M_{w}{ }^{\mathrm{b}} \\
(k D a)\end{array}$ & $\bigoplus^{\mathrm{b}}$ & $p K_{a}{ }^{\mathrm{c}}$ & $\begin{array}{c}\zeta^{\mathrm{d}} \\
(\mathrm{mV})\end{array}$ \\
\hline $\mathrm{P} 1$ & \multirow{4}{*}{$p\left(\mathrm{DEAEMA}_{m}-s t-\mathrm{MPC}_{n}\right)$} & 98 & - & 100 & 100 & 19.4 & 1.05 & 6.7 & 12.4 \\
\hline $\mathrm{P} 2$ & & 53 & 32 & 75 & 53 & 19.6 & 1.04 & - & -10.9 \\
\hline P3 & & 37 & 58 & 50 & 42 & 25.4 & 1.04 & - & -14.1 \\
\hline $\mathrm{P} 4$ & & 22 & 91 & 25 & 35 & 32.1 & 1.04 & - & -0.05 \\
\hline P5 & \multirow{4}{*}{$p\left(\mathrm{AEMA}_{m}-s t-\mathrm{MPC}_{n}\right)$} & 67 & - & 100 & 100 & 14.3 & 1.26 & 8.1 & 15.2 \\
\hline $\mathrm{P} 6$ & & 50 & 23 & 75 & 68 & 19 & 1.24 & 8 & 12.8 \\
\hline $\mathrm{P} 7$ & & 43 & 51 & 50 & 46 & 25 & 1.12 & 7.9 & 2.9 \\
\hline $\mathrm{P} 8$ & & 19 & 61 & 25 & 24 & 22.8 & 1.05 & 6.8 & 9.9 \\
\hline P9 & \multirow{4}{*}{$p\left(\mathrm{DIPAEMA}_{m}-s t-\mathrm{MPC}_{n}\right)$} & 80 & - & 100 & 100 & 18.2 & 1.05 & 5.9 & 20.8 \\
\hline P10 & & 81 & 33 & 75 & 68 & 28.2 & 1.03 & 6.4 & 5.6 \\
\hline P11 & & 65 & 64 & 50 & 56 & 35.4 & 1.07 & 6.9 & 2.2 \\
\hline $\mathrm{P} 12$ & & 22 & 91 & 25 & 29 & 32.8 & 1.02 & - & -13.8 \\
\hline P13 & \multirow{5}{*}{$p\left(\mathrm{DMAEMA}_{m^{-}}-\mathrm{MPC}_{n}\right)$} & 110 & - & 100 & 100 & 18.4 & 1.04 & 6.9 & 21.7 \\
\hline P14 & & 25 & 9 & 75 & 73 & 7.2 & 1.05 & 6.9 & 5.6 \\
\hline P15 & & 48 & 32 & 50 & 60 & 17.6 & 1.01 & 7.5 & 3.8 \\
\hline P16 & & 17 & 47 & 25 & 27 & 17.4 & 1.02 & 7.8 & 0 \\
\hline $\mathrm{P} 17$ & & - & 24 & 0 & 0 & 11.3 & 1.12 & - & -10.9 \\
\hline \multirow{3}{*}{$\begin{array}{l}\text { P18 } \\
\text { P19 } \\
\text { P20 }\end{array}$} & \multirow{3}{*}{$p\left(\right.$ DEAEMA $\left._{m}-s t-\mathrm{PEGMEMA}_{n}\right)$} & 74 & 20 & 75 & 77 & 25.6 & 1.04 & 6.9 & 4.2 \\
\hline & & 53 & 42 & 50 & 52 & 33.6 & 1.09 & 6.6 & -7.1 \\
\hline & & 36 & 65 & 25 & 33 & 43.9 & 1.06 & 6.5 & -11.7 \\
\hline \multirow{3}{*}{$\begin{array}{l}\text { P21 } \\
\text { P22 } \\
\text { P23 }\end{array}$} & \multirow{3}{*}{$p\left(\mathrm{AEMA}_{m}-s t-\mathrm{PEGMEMA}{ }_{n}\right)$} & 85 & 35 & 75 & 75 & 21.8 & 1.21 & 7.8 & 16.2 \\
\hline & & 90 & 120 & 50 & 43 & 26.8 & 1.23 & 8.1 & 8.6 \\
\hline & & 44 & 79 & 25 & 26 & 25.8 & 1.19 & 7.8 & 7 \\
\hline \multirow{3}{*}{$\begin{array}{l}\text { P24 } \\
\text { P25 } \\
\text { P26 }\end{array}$} & \multirow{3}{*}{$p\left(\right.$ DIPAEMA $\left._{m}-s t-\mathrm{PEGMEMA}_{n}\right)$} & 36 & 20 & 75 & 64 & 18.4 & 1.01 & 6.6 & 6.3 \\
\hline & & 29 & 33 & 50 & 47 & 24.4 & 1.07 & 6.8 & 2.3 \\
\hline & & 27 & 82 & 25 & 25 & 48.5 & 1.1 & 6.9 & -9.4 \\
\hline \multirow{4}{*}{$\begin{array}{l}\text { P27 } \\
\text { P28 } \\
\text { P29 } \\
\text { P30 }\end{array}$} & \multirow{4}{*}{$p\left(\mathrm{DMAEMA}_{m}-s t-\mathrm{PEGMEMA}_{n}\right)$} & 26 & 9 & 75 & 74 & 10.4 & 1.18 & 7 & 8.3 \\
\hline & & 14 & 12 & 50 & 52 & 10.6 & 1.23 & 7 & 18.5 \\
\hline & & 9 & 4 & 25 & 12 & 3.8 & 1.09 & 6.8 & -0.09 \\
\hline & & - & 7 & 0 & 0 & 6.4 & 1.77 & - & 6.8 \\
\hline \multirow{3}{*}{$\begin{array}{l}\text { P31 } \\
\text { P32 } \\
\text { P33 }\end{array}$} & \multirow{3}{*}{$p\left(\mathrm{DEAEMA}_{m}-s t-\mathrm{HEMA}_{n}\right)$} & 66 & 21 & 75 & 74 & 14.7 & 1.04 & 7.5 & 15.2 \\
\hline & & 47 & 47 & 50 & 50 & 16.1 & 1.07 & 7.6 & 9.4 \\
\hline & & 22 & 50 & 25 & 30 & 11.6 & 1.06 & 7.8 & 5.6 \\
\hline \multirow{3}{*}{$\begin{array}{l}\text { P34 } \\
\text { P35 } \\
\text { P36 }\end{array}$} & \multirow{3}{*}{$p\left(\mathrm{AEMA}_{m}-s t-\mathrm{HEMA}_{n}\right)$} & 85 & 54 & 75 & 61 & 26.2 & 1.23 & 8.2 & 22.7 \\
\hline & & 72 & 92 & 50 & 44 & 29.8 & 1.23 & 8.2 & 21 \\
\hline & & 40 & 132 & 25 & 23 & 27.1 & 1.13 & 6.9 & 18.4 \\
\hline \multirow{3}{*}{$\begin{array}{l}\text { P37 } \\
\text { P38 } \\
\text { P39 }\end{array}$} & \multirow{3}{*}{$p\left(\right.$ DIPAEMA $\left._{m}-s t-\mathrm{HEMA}_{n}\right)$} & 61 & 33 & 75 & 65 & 19 & 1.07 & 6.5 & 16.2 \\
\hline & & 52 & 50 & 50 & 51 & 20.2 & 1.13 & 7.3 & 12.8 \\
\hline & & 27 & 80 & 25 & 25 & 17.4 & 1.07 & 6.4 & -0.7 \\
\hline P40 & \multirow{4}{*}{$p\left(\right.$ DMAEMA $\left._{m}-s t-\mathrm{HEMA}_{n}\right)$} & 34 & 22 & 75 & 60 & 8.8 & 1.04 & 7.2 & 4.8 \\
\hline $\mathrm{P} 41$ & & 32 & 45 & 50 & 42 & 11.7 & 1.05 & 7.2 & 7.9 \\
\hline P42 & & 65 & 145 & 25 & 31 & 31.6 & 1.09 & 7.3 & 2.5 \\
\hline $\mathrm{P} 43$ & & - & 60 & 0 & 0 & 8.6 & 1.04 & - & 0.8 \\
\hline
\end{tabular}


(A)

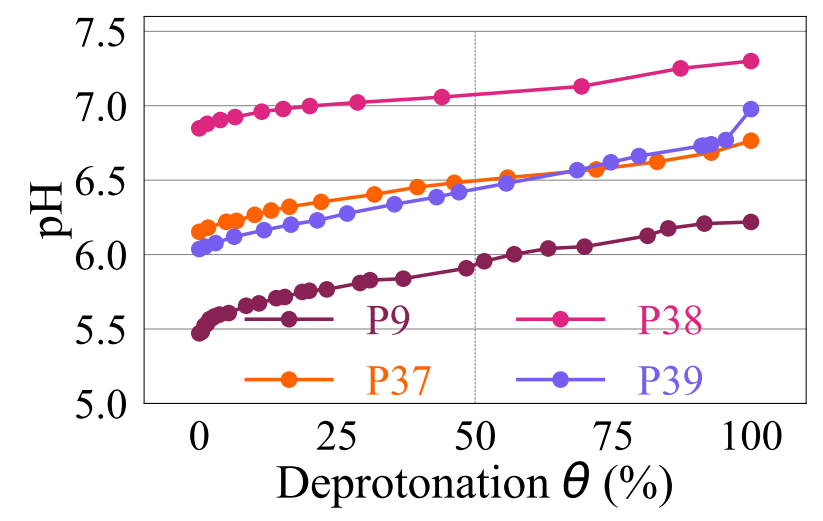

(B)

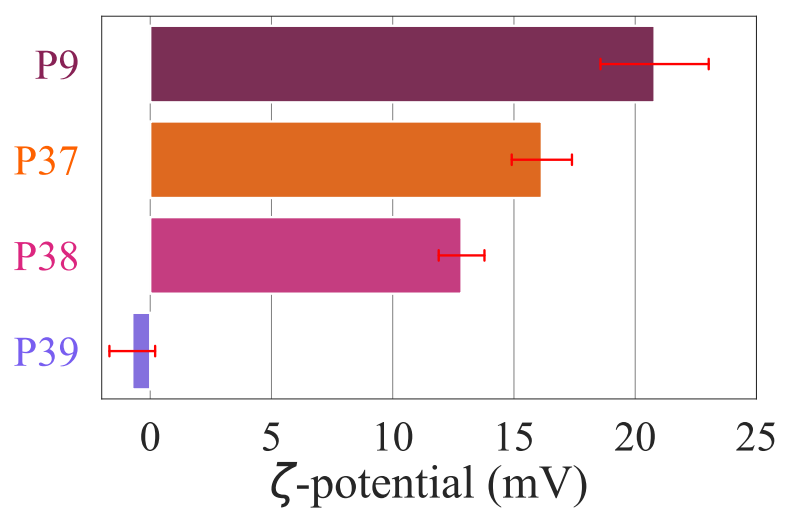

Figure 3: The effect of co-monomer incorporation on polycation protonation is illustrated through the example of the $p$ (DIPAEMA-st-HEMA) series of polymers (P9, P37-P39). (A) The $p K_{a}$ values of four polycations in this polymer sub-family were determined through potentiometric titrations. Changes in $\mathrm{pH}$ have been plotted as a function of the degree of deprotonation, $\theta$. (B) Electrophoretic mobilities of polymers were measured in PBS to monitor changes in $\zeta$-potential with decreasing cationic monomer incorporation.

A detailed characterization workflow developed to comprehensively capture the most relevant physicochemical attributes of each polymer (Table 1). We highlight this workflow by showing snapshots of data collected for the $p$ (DIPAEMA-st-HEMA) sub-family (P9, P37-P39) of copolymers (Figure 3) while data for the remaining polymers can be found in the SI. Measurements of $\zeta$-potential in PBS revealed a generalized trend; the cationic homopolymer displayed the highest charge density while the addition of HEMA repeat units gradually lowered the $\zeta$-potential to near-zero levels. Next, we examined the effect of copolymer composition on deprotonation by estimating $p K_{a}$ values via titration. The reduction in $p K_{a}$ from 8.38 to 5.9 while going from monomer to homopolymer can be attributed to the suppression of amine protonation due to electrostatic repulsion between proximate amine groups (Figures S8 and S9). In contrast, upon increasing HEMA incorporation to $25 \%, 50 \%$, and $75 \%, p K_{a}$ values increased from 5.9 to $6.5,7.3$ and 6.4 respectively. (Figure $3 \mathrm{~A})$. We attribute this non-monotonic trend in $p K_{a}$ to changes in polymer conformation, which in turn is a complex function of monomer distribution or sequence and cannot be explained by the polymer composition alone. ${ }^{46-48}$ Compared to the 
low $p K_{a}$ value of the cationic homopolymer ( $\mathrm{P} 9$ had a $p K_{a}=5.9$ ), the copolymers incorporating HEMA exhibited $p K_{a}$ values that were up to $1.4 \mathrm{pH}$ units higher, highlighting the critical, but frequently overlooked impact of polymer composition on polycation protonation. In summary, parallelized approaches to synthesis and characterization generated synthetic vector libraries that encompass a wide range of chemical compositions, interfacial properties, and protonation equilibria. This multiparametric polymer library helped us probe relationships between polymer properties, intracellular RNP delivery, cellular toxicity, and payload internalization, whilst maximizing experimental efficiency and throughput. Although larger material libraries may have been generated in previous reports, the scope of polymer characterization was severely restricted in prior studies. ${ }^{26,32-36}$ We approach the trade-off between synthetic throughput and rigor of characterization with a marked bias towards the former.

\section{Discovery of hit polymer (P38) for functional delivery of RNP payloads}

To identify structures that mediate efficient genome editing, we engineered the HEK293 cell line with the traffic light reporter (TLR) gene. ${ }^{49}$ This reporter system allows us to resolve and quantify two gene editing pathways: (1) non homologous end-joining (NHEJ), an imprecise and error-prone DNA-repair event that results in mCherry expression, (2) homology-directed repair (HDR), which proceeds through the precise insertion of repair templates (such as pDNA) and results in the expression of green fluorescent protein (GFP).

To efficiently interrogate NHEJ events mediated by polymeric vectors in our library, we developed a high-throughput assay to quantify mCherry expression. Even if 100\% NHEJ editing were to be achieved, only $33 \%$ of frameshift mutations would culminate in mCherry expression, hindering detection and quantification in instances of low editing efficiency. ${ }^{49}$ To resolve the trade-off between throughput and sensitivity, we adopted image cytometry (Figure 4A). ${ }^{50}$ Using a customized image analysis pipeline, the spatial distribution and intensity of mCherry in these fluorescence micrographs was quantified (Figure 4B). 
(A)

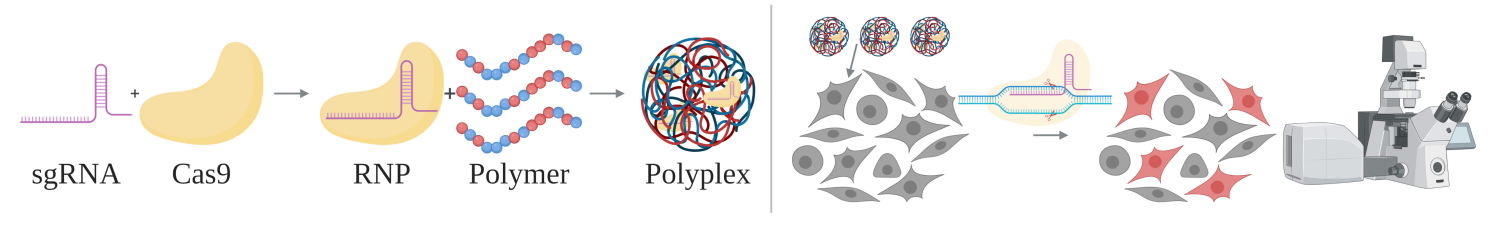

(B)

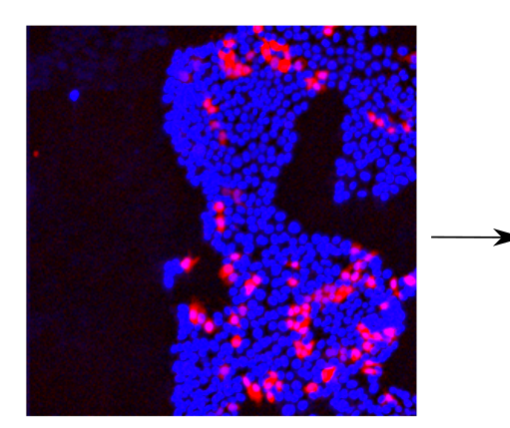

Input Image

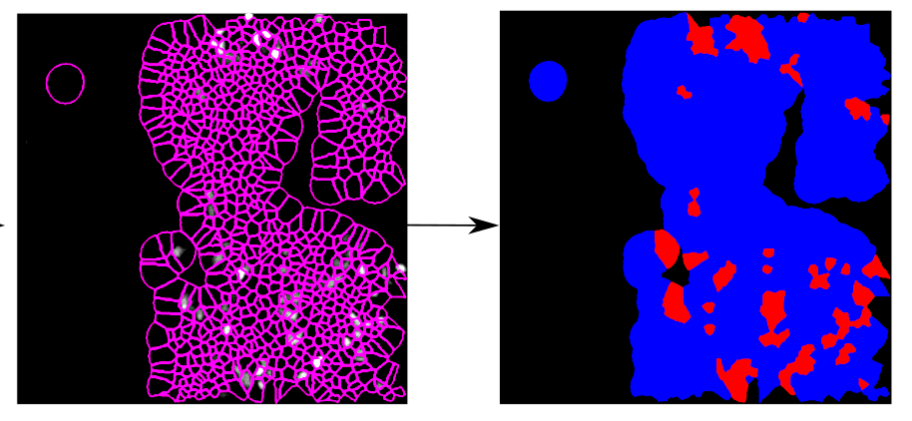

Identify cell outlines Classify mCherry $+/-$

(C)

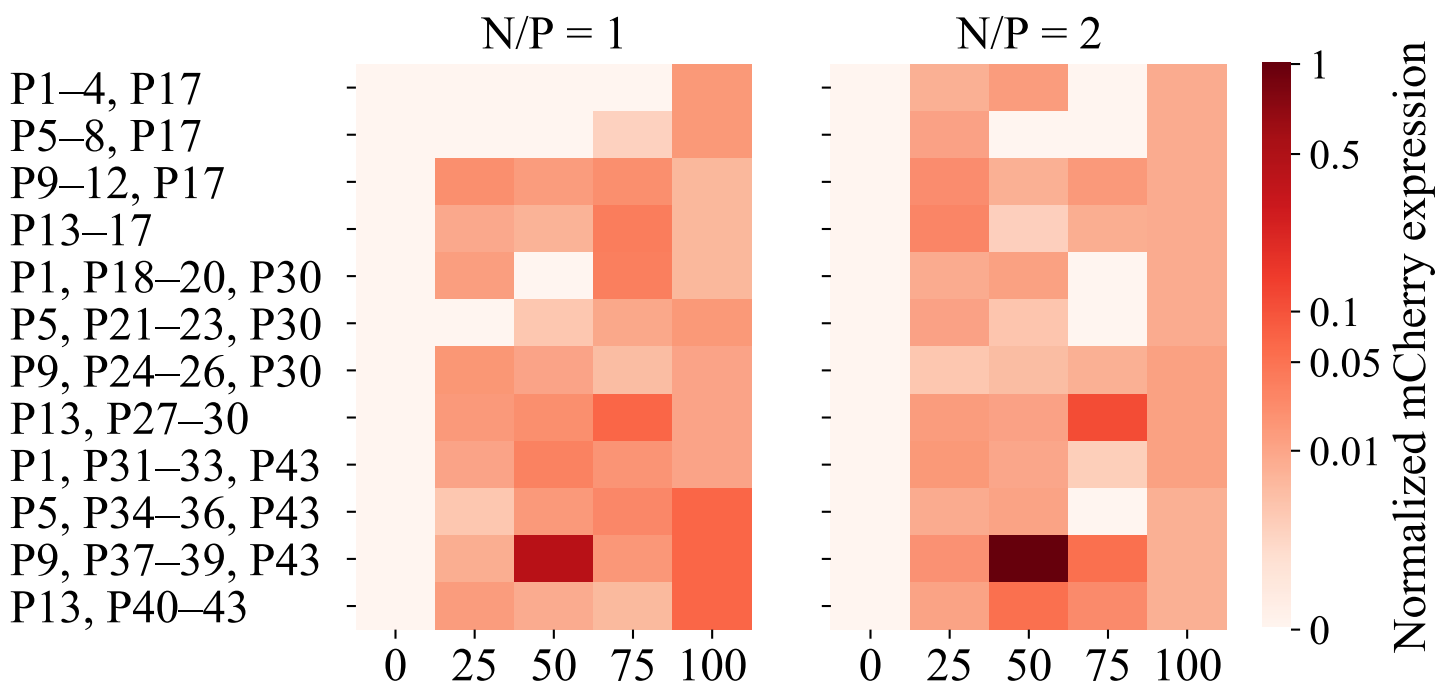

Target cationic incorporation (\%)

Figure 4: (A) Overview of screening workflow. In the HEK293 TLR cell line, if $100 \%$ editing efficiency were achieved via NHEJ, about $1 / 3 \mathrm{rd}$ of cells would produce mCherry, allowing us to adopt mCherry expression levels as an indirect measure of editing efficiency. To quantify mCherry expression and gene editing outcomes with high throughput, while ensuring adequate sensitivity to low gene editing efficiencies, image cytometry was employed in tandem with (B) high-content image analysis software pipelines. (C) RNP delivery was assessed at two N/P ratios (1 and 2) across the entire library. At the end of the screening study, P38 ( $p$ (DIPAEMA $_{52}$-st-HEMA $\left.{ }_{50}\right)$ emerged as the only hit polymer. 
To map the relationship between editing efficiencies, polymer compositions, and N/P ratios, we adopted the following procedure. First, for each polyplex formulation, mCherry expression levels were averaged across all images and normalized to the maximum value recorded in the polymer library (Figure 4C). We observed that cells treated with P38 polyplexes exhibited the highest mCherry expression levels, suggesting that this polymer achieved highly efficient intracellular RNP delivery. Excepting P38, the rest of the library resulted in either near-zero mCherry expression or marginal mCherry expression.

\section{P38 outperformed four state-of-the-art commercial vehicles, $58 \%$ editing efficiency achieved}

To benchmark P38 against commercially available lipid and PEI-based transfection reagents, we performed flow cytometric measurements. In these studies, mCherry expression resulting from NHEJ editing (Figure 5A) was quantified . RNP delivery by JetPEI and Lipofectamine 2000 resulted in $1 \%$ and $2 \%$ mCherry expression respectively, suggesting that these reagents are better suited for pDNA payloads than for RNPs. The RNP-specific reagents fared better, with both Lipofectamine CRISPRMAX (CMAX) and JetCRISPR causing around $8 \%$ of cells to express mCherry. As for P38, mCherry expression was highly dependent on the dose of polymer. At an $\mathrm{N} / \mathrm{P}$ ratio of 0.5 , editing performance was marginal, with only $2 \%$ mCherry expression, however $\mathrm{N} / \mathrm{P}$ ratios of $1,1.5$, and 2 resulted in significant improvements over commercial controls (10-12\% mCherry expression). 
(A)

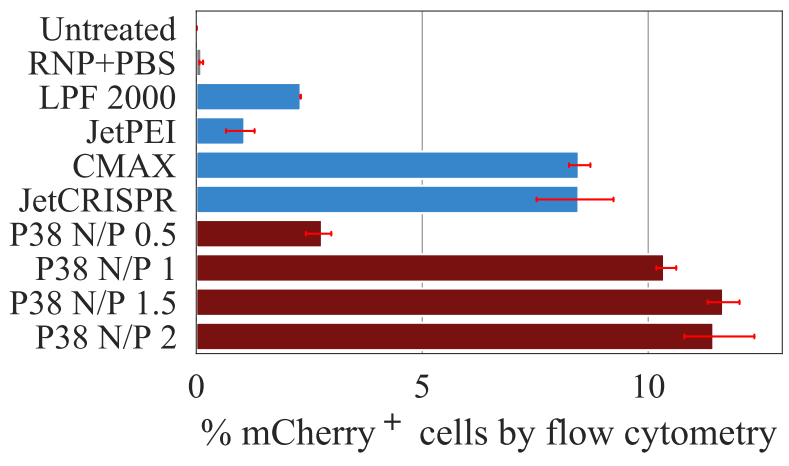

(B)

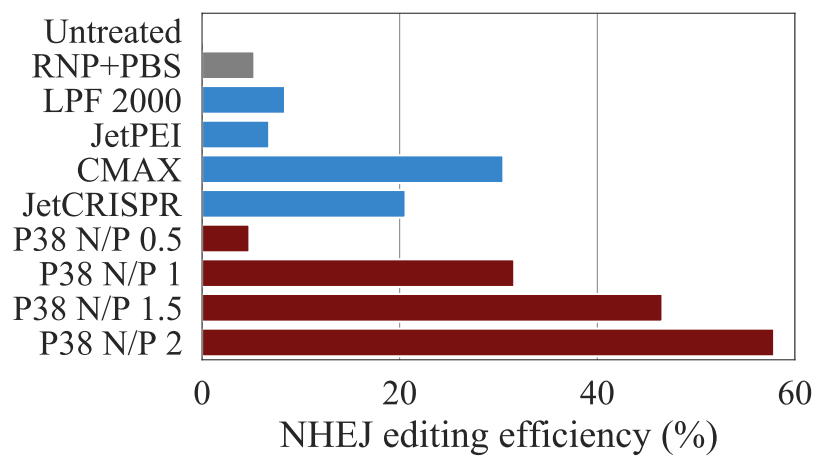

(C)

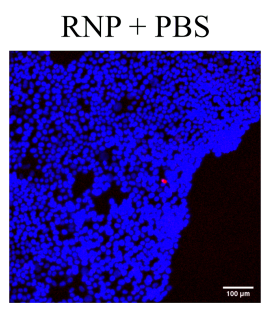

(D)
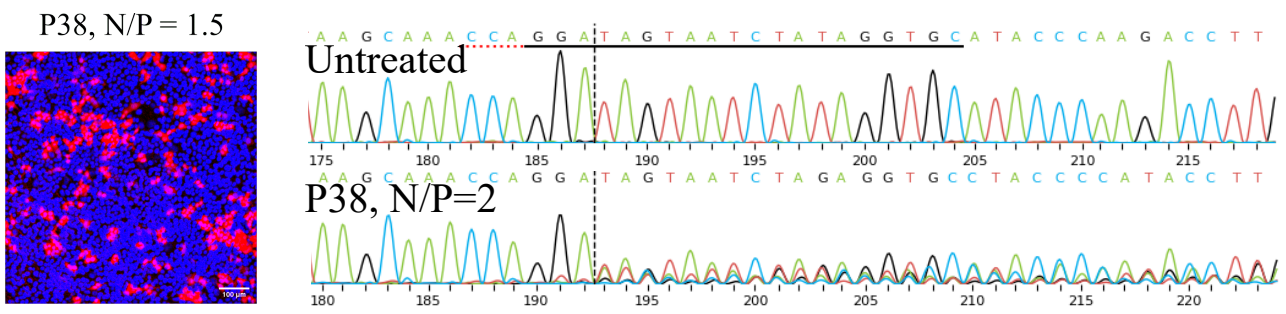

Figure 5: (A) Flow cytometry was performed to benchmark P38 against four commercial transfection reagents. At $\mathrm{N} / \mathrm{P}$ ratios of $1,1.5$, and 2, P38 resulted in higher mCherry expression than Lipofectamine CRISPRMAX (CMAX) and JetCRISPR $(\mathrm{n}=3)$. (B) NHEJ editing measured by Sanger sequencing and TIDE assay. Sanger sequencing established that P38 outperforms commercial controls, achieving 58\% editing efficiency. (C) mCherry expression in cells treated with unpackaged ribonucleoprotein and polyplexes formed with P38. Scale bar is $100 \mu \mathrm{m}$. (D) Representative chromatograms from cells treated with $\mathrm{P} 38 / \mathrm{RNP}$ polyplexes at the highest $\mathrm{N} / \mathrm{P}$ ratio.

(A)

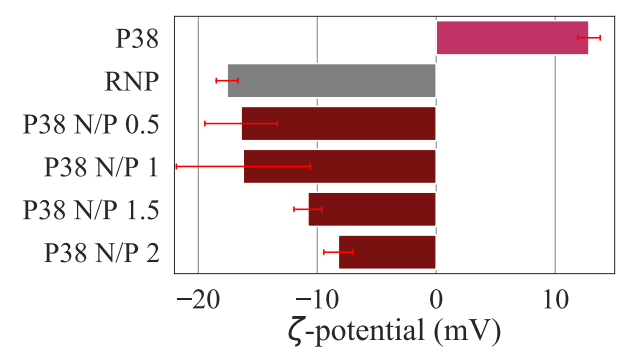

(B)

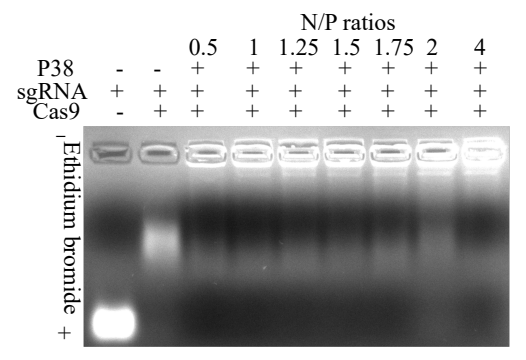

(C)

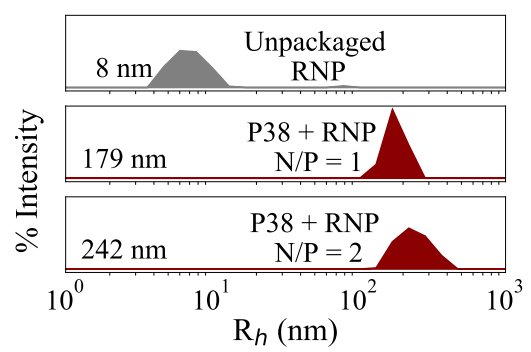

Figure 6: (A) $\zeta$-potential measurements of unpackaged RNPs and polyplexes formulated at various $\mathrm{N} / \mathrm{P}$ ratios. (B) Gel migration assays reveal small amounts of unbound $\mathrm{RNP}$ in the polyplexes, suggesting that the binding between P38 and RNPs is moderate. (C) Dynamic light scattering measurements $(\mathrm{n}=3$ to 5$)$ of RNPs and polyplexes yielded monomodal size distributions. 
In the TLR cell line, mCherry expression severely underestimates the actual editing efficiency, since only a fraction of gene disruptions results in mCherry production. Therefore, it is essential to complement flow cytometry with an analysis of the distribution of insertions and deletions (indels) culminating from NHEJ (Figure 5B). Sequencing results largely mirrored the trends observed during flow cytometric measurements, establishing that mCherry expression is a valid proxy for editing efficiency. Considering only results from commercial reagent controls, we recorded the highest editing efficiency for Lipofectamine CRISPRMAX, with $30 \%$ of the cell population containing mixed DNA sequences resulting from error-prone NHEJ. JetCRISPR reported slightly lower editing frequencies than expected (20\%) while less than $10 \%$ indel formation was observed for JetPEI and Lipofetcamine 2000. In contrast, P38-mediated RNP delivery led to editing efficiencies as high as 58\%, which is almost double that of the most efficient among commercial transfection reagents (Lipofectamine CRISPRMAX). Taken together, flow cytometry and sequencing results offer overwhelming evidence that P38 outperforms state-of-the-art commercial vehicles.

To identify the biophysical factors associated with functional RNP delivery, we performed gel migration studies and dynamic light scattering (DLS) measurements for the entire polyplex library. Since polymeric vectors must balance payload protection and rapid intracellular unpackaging, it is essential to probe the distribution of binding states among polymer-RNP complexes using gel electrophoresis assays. In the case of P38, we observed that RNPs existed in two binding configurations: polymer-bound RNPs that did not migrate in response to the applied electric field and a small population of RNPs that migrated (Figure 6B) to the positive electrode. To gain further insight into polymer-RNP association, $\zeta$-potential measurements of polyplexes were performed (Figure 6A). Since P38 has a $p K_{a}$ of 7.3 , we expected roughly half the tertiary amines along the polymer backbone to exist in a protonated state at physiological $\mathrm{pH}$, giving rise to a net positive charge (12.8 $\mathrm{mV}$ in PBS). The spCas9 protein is known to possess an electrostatically heterogeneous 
surface, while the sgRNA has an anionic backbone. ${ }^{51}$ As a result, the RNP complex bears a charge of $-17.5 \mathrm{mV}$ in $\mathrm{PBS}$. Among polyplex formulations with $\mathrm{N} / \mathrm{P}$ ratios of 0.5 and 1 , the extent of negative charge was reduced, but polarity was not reversed. Surprisingly, charge inversion was not achieved even at higher $\mathrm{N} / \mathrm{P}$ ratios of 1.5 and 2 , although their negative surface potentials were reduced in magnitude. Our unexpected observation of net negative charge may be explained by the presence of free RNPs co-existing with weakly bound RNP-polymer complexes, mirroring findings from gel migration assays. Given the high delivery efficiency of P38, it appears that the formation of tightly bound polymer-RNP complexes is not essential for ensuring efficient RNP delivery.

Since membrane association, endocytosis, and intracellular trafficking are all size-sensitive phenomena, we performed DLS measurements for the entire library (Figures S9, S10, and S11). As for P38, RNPs formulated at N/P ratios of one and two were around $180 \mathrm{~nm}$ and $240 \mathrm{~nm}$ in hydrodynamic radius, which is around thirty times the size of the unpackaged RNP (Figure 6C). The tendency of the hit formulation to form polyplexes larger than $100 \mathrm{~nm}$ in radius, has interesting implications for cellular internalization and nuclear accumulation. ${ }^{52}$ While particles smaller than $100 \mathrm{~nm}$ in radius exhibit a preference for clathrin-mediated pathways, larger particles are internalized via caveolar pathways, which permit polyplexes to traverse the cytosol and enter the nucleus while avoiding lysosomal interrogation. ${ }^{19,53}$ In addition, physical aspects of bolus transfection cannot be overlooked: upon introduction into the cell culture media, bulkier polyplexes will reach the surface of adherent monolayer cultures faster than smaller polyplexes. ${ }^{54}$ The large size of P38 polyplexes may have eliminated the need to improvise endosomal escape routes and imparted favorable transport characteristics that maximized polyplex-cell contact.

Herein we discovered that: (1) binding between the RNP payload and the polymer was incomplete, with a small fraction of RNPs remaining unbound, (2) although the polyplex size exceeds the optimal range for in vitro delivery, P38 was somehow able to direct RNP payloads along cellular pathways favoring nuclear uptake. Despite these departures from 
the expected design rules, P38 proved to be an excellent chemically defined vector, with a delivery efficiency far higher than that of commercially available reagents, illustrating the utility and power of the high-throughput approach to deliver efficient polymeric vehicles from under-explored domains in chemical space.

\section{Cytotoxicity, cellular uptake, and intracellular payload distribution}

(A)

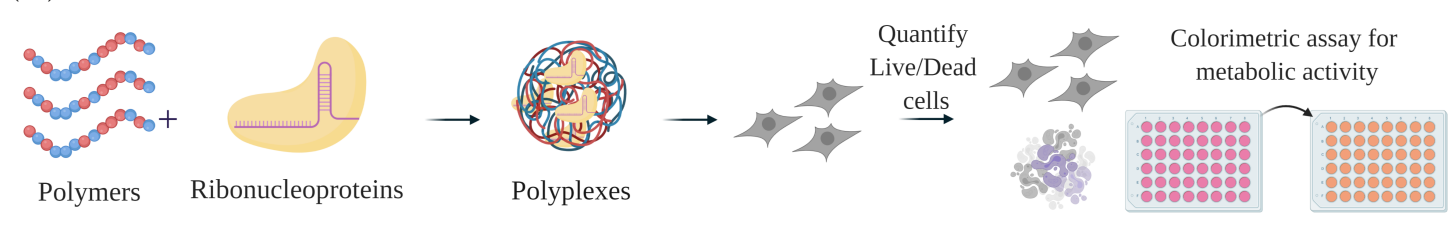

(B)

P1-4, P17
P5-8, P17
P9-12, P17
P13-17
P1, P18-20, P30
P5, P21-23, P30
P9, P24-26, P30
P13, P27-30
P1, P31-33, P43
P5, P34-36, P43
P9, P37-39, P43
P13, P40-43
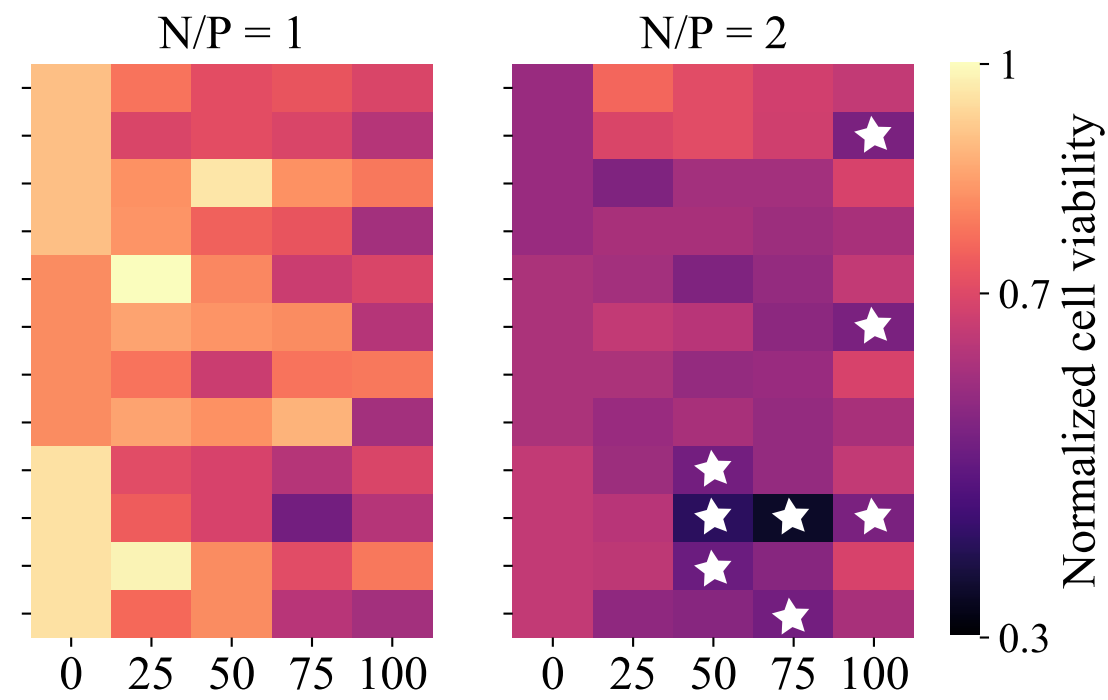

Target cationic incorporation (\%)

Figure 7: (A) Cytotoxicity measurements employed the CCK-8 assay (B) Heatmap of normalized cellular viability as a function of polymer composition and $\mathrm{N} / \mathrm{P}$ ratio. Formulations with the lowest cellular viability (bottom 5 percentile) are highlighted with white stars. Six polymers, P5 (highlighted in all three instances), P32, P34, P35, the hit polymer P38, and P40 were identified as the most toxic. Except for P5 (the homopolymer of AEMA), all of them incorporate HEMA as a co-monomer.

Combinatorial polymer design and parallelized experimentation rapidly yielded a promising lead structure (P38) that exceeds benchmarks set by commercial PEI and 
lipid-based reagents for RNP delivery. Yet, our inability to identify any obvious structural driver of P38's intracellular delivery efficacy prompted us to expand the scope of our investigations beyond editing efficiency to include toxicity and the cellular internalization of RNP payloads.

First, we studied how the cellular toxicity originating from polyplex-mediated RNP delivery is shaped by the identity and the degree of incorporation of hydrophilic co-monomers, the $p K_{a}$ of the cationic monomer, N/P ratios, polymer $p K_{a}$, charge densities, and polymer hydrophobicity. We measured the toxicity associated with each polyplex formulation through CCK-8 assays and plotted responses in the form of a heat map (Figure 7). For most cationic monomers, increasing cationic monomer incorporation was accompanied by higher cytotoxicity, although the relationship between cationic monomer incorporation and toxicity was not always monotonic. We were surprised to discover that P38 was not the only polymer in library to result in deterioration of cellular viability. Five other polymers (highlighted with stars in Figure 7) induced comparable or higher levels of toxicity than P38. Excepting P38, each of these formulations were ineffective at mediating functional RNP delivery during image cytometry screening. In this list of high-toxicity formulations, except for P5 (a homopolymer of AEMA), all polymers were constituted from HEMA, the least hydrophilic co-monomer in the library. This underscores the importance of co-monomer hydrophilicity and phase behavior in shaping cell-polyplex interactions. 
(A)

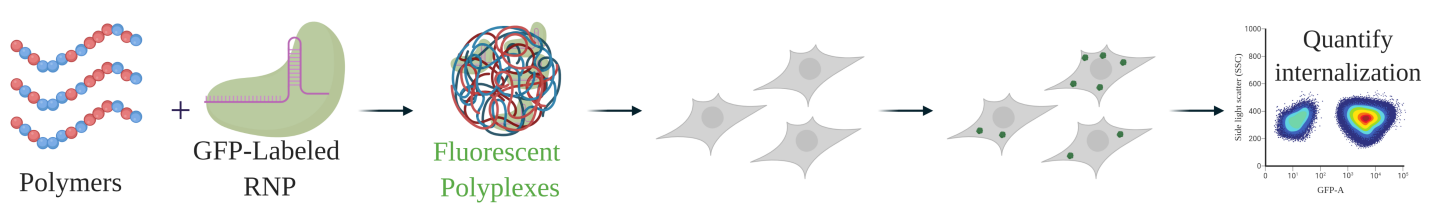

(B)

P1-4, P17
P5-8, P17
P9-12, P17
P13-17
P1, P18-20, P30
P5, P21-23, P30
P9, P24-26, P30
P13, P27-30
P1, P31-33, P43
P5, P34-36, P43
P9, P37-39, P43
P13, P40-43
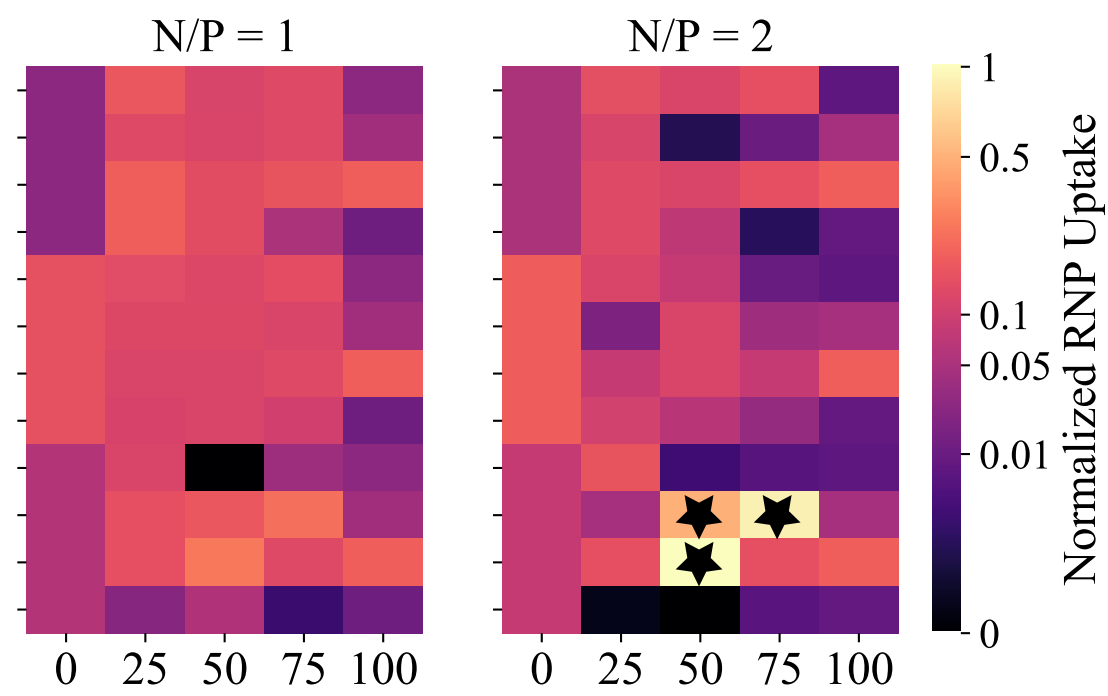

Target cationic incorporation $(\%)$

Figure 8: (A) GFP-labeled RNPs were complexed with each polymer at two N/P ratios. Flow cytometry was used to measure polyplex uptake across the entire library (B) Cellular uptake of RNPs (geometric mean of GFP intensity was normalized) represented as a function of polymer composition and $\mathrm{N} / \mathrm{P}$ ratio. Instances of highly efficient internalization are highlighted with black stars. The hit polymer, P38 as well as two copolymers from the $p$ (AEMA-st-HEMA) series (P34 and P35) displayed the highest cellular uptake.

Next, we investigated the relationship between RNP uptake and polyplex composition through a library-wide internalization study. We recognized that while several polyplexes may be limited by low cellular internalization, for some other polyplex formulations, endolysosomal navigation and payload unpackaging might have prohibited RNP release within the cytosol. By measuring RNP internalization efficiency, we hoped to discriminate between these two failure modes: low cellular uptake and intracellular payload release. Annealing sgRNA with a fusion protein of GFP and spCas9, we generated fluorescent RNP variants to quantify the RNP internalization effected by forty-three polymers in the library 
at two $\mathrm{N} / \mathrm{P}$ ratios (Figure $8 \mathrm{~A}$ ). As expected, P38 polyplexes were internalized most efficiently at both $\mathrm{N} / \mathrm{P}$ ratios studied, with the majority of the polyplex library resulting in RNP uptake levels comparable to those achieved by uncomplexed or naked payloads (Figure 8B). Surprisingly, two copolymers from the $p$ (AEMA-st-HEMA) sub-family (P34 and P35) gave rise to RNP uptake levels comparable to those of P38 (highlighted in the heatmap with black stars). Excepting these three structures (P34, P35, and P38), interactions between polyplexes and cell membranes did not culminate in cellular entry, explaining the sub-par editing efficiencies that were observed for the majority of the library. Unlike other underperforming polymer candidates, the RNP delivery of P34 and P35 was not compromised by inefficient cellular internalization; rather we suspected that the intracellular trajectories of these two polyplex formulations did mirror that of P38, consigning them to a different fate.

To examine the subcellular localization of polyplexes, we performed immunofluorescence studies with GFP-labeled RNPs and anti-LAMP2 antibodies that facilitated visualization of lysosomal compartments (Figure 9). We compared the intracellular distribution of labeled RNPs complexed with the hit polymer P38, and the two near-miss polymers, P34 and P35 at an $\mathrm{N} / \mathrm{P}$ ratio of two. We observed that all three sets of polyplexes were successfully internalized, as indicated by the appearance of GFP signals associated with the RNPs. We performed Z-stacked confocal scans (four replicates) for these three treatment groups and quantified the Pearson's correlation coefficient (PCC) between the GFP signals from labeled RNPs and the red signals from labeled lysosomes using Costes' method. ${ }^{55}$ The mean PCC was highest $(0.71 \pm 0.29)$ in P34 and lowest in the hit polymer P38 (0.13 \pm 0.11$)$ while P35 had a PCC of $0.51 \pm 0.33$. From colocalization analysis (Figure S25), we found that nearmiss polymers, P34 and P35 were more likely to co-localize with lysosomal compartments than the hit polymer P38. 

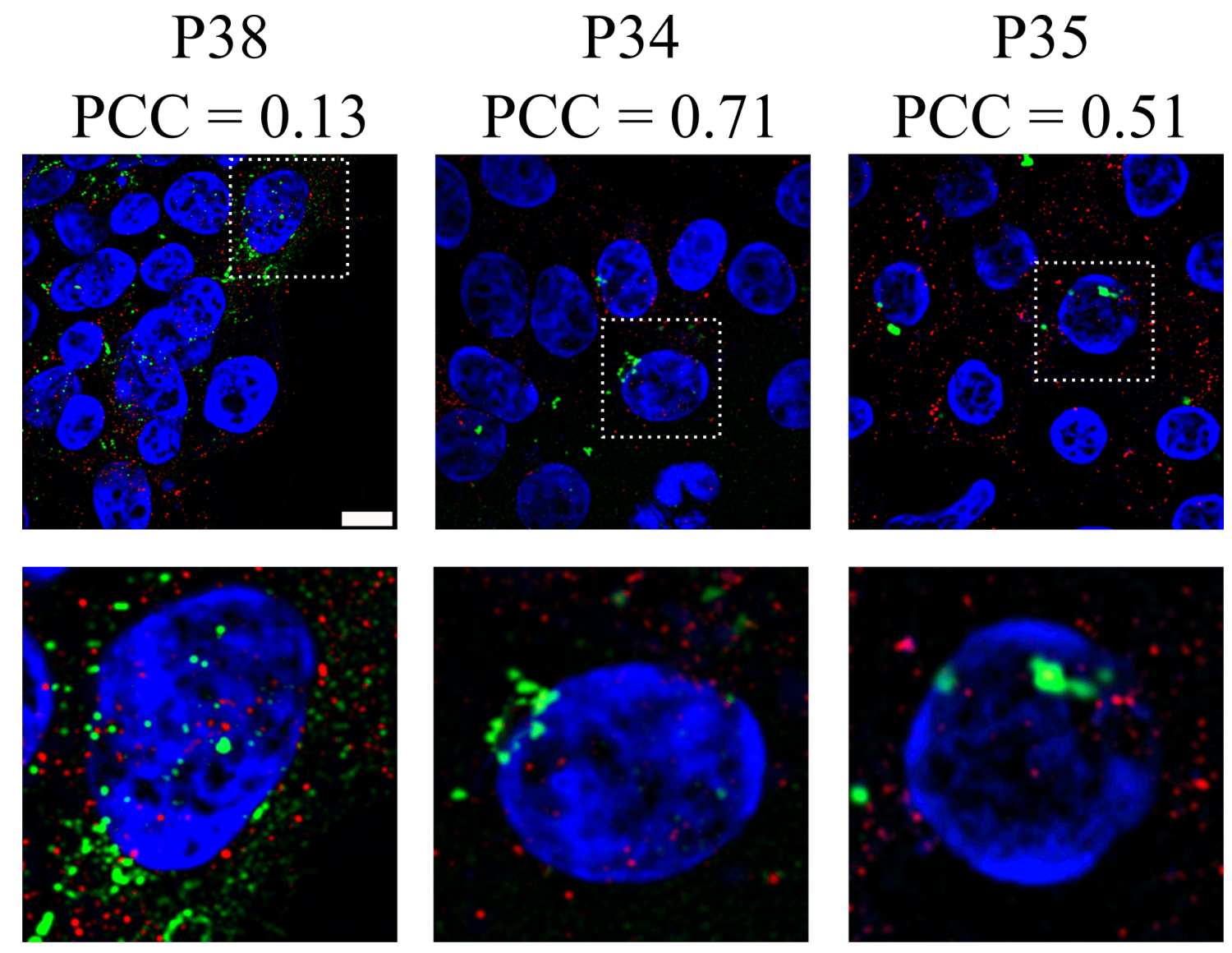

Figure 9: Representative images, depicting the intracellular distribution of GFP-labeled RNP payloads for the hit polymer P38, as well as the "near miss" polymers, P34 and P35. Lysosomal compartments are visualized in the red channel and nuclei in the blue channel. Scale bar is $10 \mu \mathrm{m}$.

Statistical learning uncovers structural drivers of cytotoxicity, cellular uptake, and editing efficiency.

From our library-wide evaluation of toxicity and RNP internalization, we discovered that P38 was neither the sole polymer to effect high cellular internalization of RNP payloads nor unique in perturbing cellular health. And yet, P38 alone achieved efficient genome editing, suggesting that the structural determinants underlying delivery, toxicity and internalization are not identical. We turned to data science techniques to elucidate the surprising contrasts in delivery performance between P38 and the rest of the library. Did a single dominant design attribute mark P38 out for success or did multiple polyplex attributes act in concert? 
What are the design attributes shared by P38 and the near-miss polymers that resulted in high RNP uptake? Why did our near-miss polymers exhibit cellular internalization rate comparable to P38 and yet fail to deliver RNP payloads efficiently? Since intuition-based methods of pattern recognition failed to answer these questions, we applied random forest classifiers (RFC) on datasets comprising measurements of RNP delivery, toxicity, and uptake.

We included 9 polymer descriptors in our analysis, of which 7 were derived routinely: polymer composition (abbreviated as \% cat.), polymer length $\left(M_{n}\right)$, the $\mathrm{N} / \mathrm{P}$ ratio, the $p K_{a}$ (Figure S10) and $\zeta$-potential values of the polymers, the polyplex hydrodynamic radius $\left(R_{h}\right.$, Figures S12-S14), and qualitatively determined RNP binding affinities (Figures S15-S17). To account for hydrophobicity, we employed fragment-based approaches ${ }^{56,57}$ to estimate the octanol-water partition coefficients of oligomeric approximations (10-mers) of our polymers through ClogP calculations. Finally, we quantified the cooperativity during hydrophobicallydriven polymer deprotonation by computing the Hill coefficient $\left(n_{\text {Hill }}\right)$ from $p K_{a}$ titration curves using the approach reported by Li et al. ${ }^{58}$ (Figure S11).
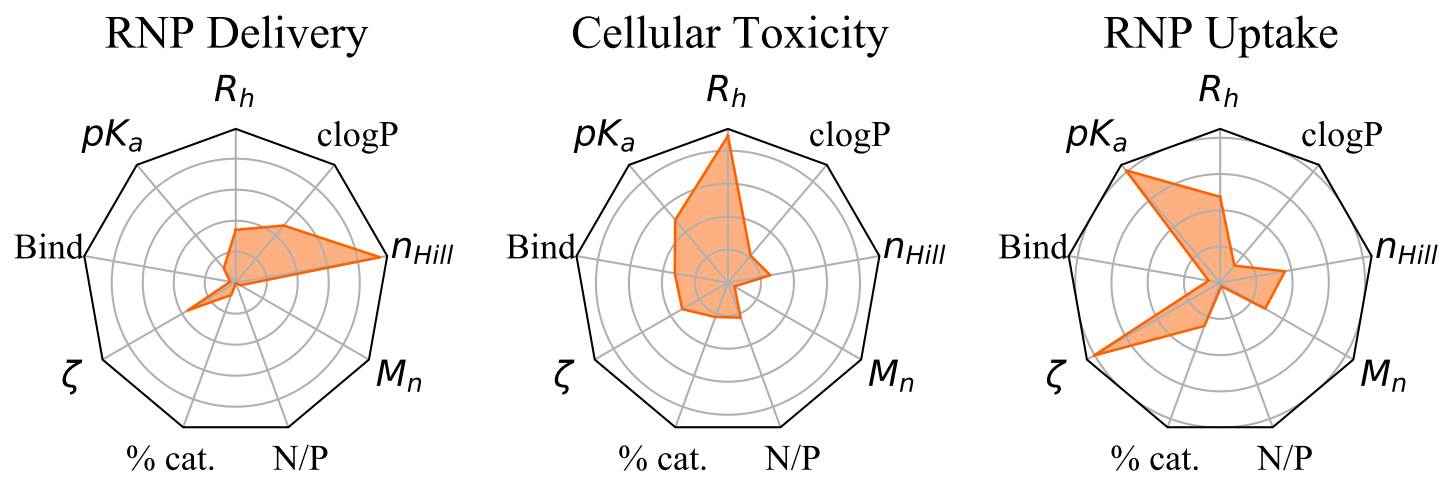

Figure 10: Random forest classifiers (RFCs) were used to map nine physicochemical descriptors (polyplex radius, polymer hydrophobicity, the Hill coefficient that parametrizes cooperative deprotonation, polymer length, N/P ratio, polymer composition, charge density, RNP binding affinity and the $p K_{a}$.) to three key biological responses (RNP delivery, cell viability, and RNP internalization). While editing efficiency is highly dependent on hydrophobicity-associated parameters such as $\operatorname{cog} \mathrm{P}$ and $n_{H i l l}$, toxicity is driven by polyplex diameter and protonation-dependent parameters such as $\zeta$-potential, RNP binding and $p K_{a}$.

Three random forest classifiers (RFCs) were trained on the RNP delivery, toxicity, and 
RNP uptake datasets, respectively. To build RFCs, we constructed ensembles of "decision trees", wherein each decision tree examines small cross-sections of the dataset using randomly selected subsets of the 9 physicochemical descriptors to classify each polymer according to the criteria specified: : (1) not a hit/hit (normalized mCherry $>0.4$ ), (2) less toxic/toxic (criterion: bottom 5 percentile of cellular viability), and 3) low/high RNP uptake ( $>30 \%$ of the mean GFP fluorescent intensity observed with the lead structure P38). Randomization of both data points and descriptors ensures that the classification rules developed by each decision tree are diverse, that multiple explanations are considered, and that we obtain a representative picture of the underlying patterns in the data. By quantifying the reliance of decision trees on these nine descriptors during classification tasks, we calculated the feature importance of each descriptor in determining RNP delivery, cellular toxicity, and RNP uptake.

We visualized feature importance across these three biological readouts in the form of a radar plot (Figure 10) and observed striking differences. Hydrophobicity-linked parameters such as $\operatorname{cog} \mathrm{P}$ and $n_{\text {Hill }}$ were the primary structural drivers of efficient RNP delivery while polyplex size $\left(R_{h}\right)$ and polymer charge density ( $\zeta$-potential) also contributed marginally. Although hydrophobicity enhancement has frequently been touted as a potent design strategy essential to achieve efficient polymeric gene delivery, ${ }^{23,59-62}$ no study has previously examined the role of hydrophobically driven cooperative deprotonation in enhancing nucleic acid delivery. Wu et al. ${ }^{63}$ recently reported that chemotherapeutic drug combinations with elevated dose response Hill coefficients displayed the highest anti-tumoral activity. ${ }^{63} \mathrm{Li}$ et al. ${ }^{58}$ demonstrated that when polymer hydrophobicity exceeds a certain threshold, phase separation caused by hydrophobic collapse of deprotonated polymer segments drives further deprotonation, thereby engineering a highly cooperative deprotonation process and leading to polymers with high Hill coefficients. We speculate that polymers such as P38, which are characterized by high $n_{\text {Hill }}$ values, unpackage their payloads more rapidly within the cytosol, compared to those with low 
$n_{\text {Hill }}$. In the case of cellular toxicity and RNP uptake, clogP and $n_{\text {Hill }}$ had negligible impact. Polyplex diameter was the most influential structural determinant of toxicity, with bulkier polyplexes causing more severe toxicity. We attributed the size-dependence of polyplex-mediated cytotoxicity to higher frequencies of polyplex-cell contacts resulting from faster settling velocities of bulkier polyplexes during bolus transfection. ${ }^{64}$ As for RNP uptake, polymers with higher values of $p K_{a}$ and $\zeta$-potential were more adept at gaining cellular entry, possibly through electrostatically mediated interactions with the negatively charged cell membrane. This finding is consistent with previous reports that elevated surface charge density and high degree of protonation can promote non-specific cellular uptake of polyplexes. ${ }^{65-69}$

Although optimizing protonation equilibria to increase the $p K_{a}$ and maximizing polycation charge density (to boost the $\zeta$-potential) may promote high cellular uptake among polymers such as P34 and P35, such structures will prove to be ineffectual in releasing payloads within the cytosol if they don't satisfy the design constraints for clogP and $n_{H i l l}$. To mediate efficient intracellular unpackaging, we must maximize $\operatorname{cog} \mathrm{P}$ and the Hill coefficient, by incorporating co-monomers of moderate hydrophilicity and tertiary amines with bulky hydrophobic substituents, while avoiding extremely hydrophilic monomers such as PEG and zwitterionic moieties such as phosphoryl choline.

\section{Conclusions}

We adopted parallelized experimental workflows and combinatorial polymer design to efficiently explore a multivariate polycation design space. Combinatorial design allowed us to create a chemically diverse polymer library and access a wide range of polymer physicochemical properties. Through parallelized experimentation, we acquired an extensive suite of physicochemical characterization data consisting of polymer composition, molecular weight distribution, $p K_{a}$, polyplex size distribution, RNP binding affinity, charge 
density, as well as key biological readouts such as cellular toxicity, RNP uptake, and RNP delivery efficiency. Image cytometry identified a hit polymer within this chemical space (P38), which outperformed all four commercial RNP delivery reagents that it was benchmarked against. Sanger sequencing was applied to analyze indel formation during NHEJ editing and these unambiguous estimates of editing efficiency validated our conclusions from image cytometry. With an editing efficiency of 58\%, which is twice that of JetCRISPR and Lipofectamine CRISPRMAX, P38 is an exciting prospect for non-viral ex vivo gene editing. To identify the physicochemical basis underlying the RNP delivery capabilities of P38, we examined correlations between polymer structure, properties and biological performance. A library-wide evaluation of editing efficiency, cellular viability, and internalization formed the basis for statistical learning models that captured key biophysical trends. Although polyplex aggregation and polycation protonation equilibria drive cellular toxicity and RNP uptake respectively, RNP unpackaging, and functional intracellular delivery will be achieved only by engineering hydrophobic polycations that deprotonate cooperatively. Combinatorial polymer design and parallelized experimentation not only streamlined the synthesis, characterization, and testing of polymeric vectors, but also delivered a promising hit polymer that may have otherwise eluded chemical intuition. We applied data science tools on this high-dimensional dataset to identify physicochemical determinants of payload delivery, cellular uptake, and toxicity, thereby providing polymer chemists with valuable experimental guidance to tailor vector properties to meet varied clinical objectives. By extending this powerful methodology across diverse cell types and nucleic acid modalities, we will establish an efficient discovery pipeline for synthetic vectors whose properties can be tailored on demand to fulfill a multitude of therapeutic needs. 


\section{Methods \& Materials}

\section{Polymer synthesis \& purification}

All reagents were purchased from Sigma Aldrich except for 4-cyano-4-[(ethylsulfanylthiocarbonyl) sulfanyl] pentanoic acid (CEP), which was purchased from Asta Tech (China), and used without further purification. RAFT polymerization was performed in the Carousel 12 parallel synthesizer (Radleys, UK) which enables the completion of twelve RAFT reactions in parallel. The quantities of chain transfer agent (CTA), initiator, monomers, and the solvents dispensed are specified in Table S1. After adding desired quantities of monomer, CTA, initiator, and solvent, all reaction modules were simultaneously degassed using 3-4 freeze-pump-thaw cycles, heated to a temperature of $78{ }^{\circ} \mathrm{C}$, and stirred overnight under an inert $\mathrm{N}_{2}$ environment. The next morning, the reaction mass was quenched and an equal volume of $1 \mathrm{~N} \mathrm{HCl}$ added. Polymer purification was performed through dialysis. Dialysis membranes with a molecular weight cut off of 3000 Da (Spectrum Chemicals, NJ) were used. Polymers were dialyzed over three days in acidified water that was replaced twice daily. Finally, lyophilization (SP Scientific, PA) was performed for two days, yielding pure polymers.

\section{Polymer characterization}

NMR was performed on the Bruker Avance III HD 500 instrument. A total of thirty-two scans were acquired using a relaxation time of ten seconds. Size exclusion chromatography (Agilent, CA) was performed using refractive index and multiple angle light scattering detectors (Wyatt, Santa Barbara, CA) to determine the complete molecular weight distribution for all copolymers. An automated titrator, Orion star T901 pH titrator (Thermo Fisher, Waltham, MA) was employed for $p K_{a}$ determination, with an initial polymer concentration of $0.5 \mathrm{mg} / \mathrm{mL}$. The Zetasizer (Malvern Instruments, MA) was used to evaluate the $\zeta$-potential of polymers dissolved in PBS, with three to five replicates each. 


\section{Physical characterization of polyplexes}

Synthetic single guide RNA (100 bp) was synthesized with a sequence of GCACCUAUAGAUUACUAUCCGUUUUAGAGCUAGAAAUAGCAAGUUAAAAUAAGG CUAGUCCGUUAUCAACUUGAAAAAGUGGCACCGAGUCGGUGCUUUU (Synthego, CA). The spCas9 protein was ordered from Aldveron, ND. Polymer stock solutions were prepared in PBS at a concentration of $1 \mathrm{mg} / \mathrm{mL}$, sterile-filtered, and further diluted in PBS to achieve targeted $\mathrm{N} / \mathrm{P}$ ratios. Ribonucleoproteins were assembled by adding sgRNA $(0.039 \mathrm{mg} / \mathrm{mL}$ and $0.05 \mathrm{mg} / \mathrm{mL}$ for DLS and gel migration respectively) to equal volumes of Cas9 protein $(0.19 \mathrm{mg} / \mathrm{mL}$ and $0.25 \mathrm{mg} / \mathrm{mL}$ for DLS and gel migration respectively), obtaining a 1:1 molar mixture. Both sgRNA and spCas9 stock solutions were diluted in BPS to obtain desired concentrations. RNP complexes were annealed for 10-15 min at room temperature. Then, equal volumes of diluted polymer solutions were added to form polyplexes. Polyplexes were maintained at ambient temperature for 45 minutes prior to DLS or gel migration studies.

Gel casting was completed using $1.5 \% \mathrm{w} / \mathrm{v}$ agarose solutions in TAE buffer. To visualize sgRNA bands, ethidium bromide was used at a concentration of $0.017 \% \mathrm{v} / \mathrm{v}$. Electrophoresis was performed at $80 \mathrm{~V}$ over 60 minutes and imaged using a transilluminator (Fotodyne, IL) under UV light. For DLS, the DynaPro plate reader III (Wyatt Instruments, CA) was used and three to five replicates were performed. For electrokinetic characterization of polyplexes, the sgRNA concentration was fixed at $0.05 \mathrm{mg} / \mathrm{mL}$ and the spCas9 concentration at 0.25 $\mathrm{mg} / \mathrm{mL}$. Three to five measurements were collected per sample using the Zetasizer (Malvern Instruments, MA).

\section{Biological assays}

The HEK293 cell line engineered with traffic light reporter system ${ }^{49}$ was used to assess RNP delivery. To obtain a stable cell line, sub-cloning was performed at the Genome Engineering Shared Resource (Minneapolis, MN). Cells were maintained in DMEM 
supplemented with $10 \%$ heat-inactivated fetal bovine serum (Thermo Fisher, Waltham, MA) at $37{ }^{\circ} \mathrm{C}$ and $5 \% \mathrm{CO}_{2}$ in $75 \mathrm{~cm}^{2}$ cell culture flasks. All polymer stock solutions were formulated at a concentration of $1 \mathrm{mg} / \mathrm{mL}$ and sterile filtered. The spCas9 protein (Aldevron, ND,) and sgRNA (Synthego, CA) solutions were prepared at concentrations of $0.039 \mathrm{mg} / \mathrm{mL}$ and $0.19 \mathrm{mg} / \mathrm{mL}$ respectively in PBS. Ribonucleoproteins were formed by addition of sgRNA to spCas9 and annealing for 15 minutes. Thereafter equal volumes of diluted polymer solutions were added to the RNP to achieve desired N/P ratios. Polyplexes were diluted in OptiMEM before introducing them to the cells. For 24-well plates, a nucleic acid loading of $1 \mu \mathrm{g} / \mathrm{mL}$ sgRNA was employed. Manufacturer's protocols were executed for commercial reagents. Image cytometry was performed in FluorBrite (Thermo Fisher, Waltham, MA) and cells were stained by adding 1 drop Nucelobrite (Thermo Fisher, Waltham, MA) to each well to visualize nuclear outlines and facilitate cell counting. Live cell imaging was performed using the Zeiss Cell Observer (Zeiss AG, Switzerland) equipped with a motorized stage, environmental control chamber, and automated image acquisition features. Images were analyzed using Cell profiler using previously detailed procedures. ${ }^{64}$

For flow cytometric measurements of mCherry, cells were stained with a Calcein violet viability marker and the $405 \mathrm{~nm}$ and $560 \mathrm{~nm}$ laser lines were used on the ZE5 (Biorad Inc, CA). Single live cells were used for analysis and gating schemes are furnished in Figure S21. At least 80,000 events were collected for every treatment condition and three technical replicates were performed. For sequencing, the DNA of transfected cells was extracted using QuickExtract DNA extraction solution (Lucigen, WI), according to the manufacturer's protocol. PCR amplification was completed using the AccuPrime Taq DNA Polymerase kit (Thermo Fisher, Waltham, MA), in accordance with the PCR recipe recommended by the manufacturer. Primer sequences used were 5' AGACCACCCCCATGTACAAA 3' and 5' GGaAaACCCTTCCTGGTTtC 3'. Primers were ordered from Integrated DNA Technologies (IDT, Skokie, IL) and dissolved in ultrapure water. Subsequent to PCR, amplified products were purified using gel 
electrophoresis in 1 wt \% agarose gel . Excised gel fragments were further purified using Monarch DNA gel extraction kit (New England BioLabs, MA). Purified DNA products were eluted in ultrapure water and analyzed using Sanger sequencing (University of Minnesota Genomics Center, Twin Cities Campus) after the addition of primer.

For cytotoxicity, cell culture media was replaced with a $2 \%$ solution of CCK- 8 (Dojindo) in Fluorbrite two days after transfection. Thereafter, cells were incubated for four hours and absorbance measured at $450 \mathrm{~nm}$ using the Synergy H1 plate reader (Biotek, CA). Measurements of the CCK-8 solution placed in empty wells were collected and this blank reading was subtracted from all data points. Absorbance values were normalized to untreated cells. Six wells were employed per treatment group. For uptake studies, GFP-labeled Cas9 (Thermo Fisher catalog\# CAS9GFPPRO-250UG) was used to formulate RNPs. Twenty-four hours after polyplexes were administered, cells were incubated with CellScrub (Genlantis, San Diego, CA) at room temperature for ten minutes and washed again with PBS to remove extracellularly bound RNPs. For confocal imaging, cells were plated on sterilized gelatin-coated glass cover slips in 24-well plates a day before transfection. A day after transfection, cells were fixed via immersion fixation and immunofluorescent labeling of lysosomes was completed with the anti-LAMP2 primary antibody (Abcam catalog\# ab25631, Cambridge, MA) and a secondary antibody (Invitrogen catalog\# A11003) diluted to 1:200 and 1:1000 respectively. Antibodies were diluted in a solution of PBS containing $5 \%$ bovine serum albumin, $0.2 \%$ gelatin, and $0.1 \%$ Triton-X. Cells were counterstained with Hoechst 3342 and three washing steps of five minutes each were performed with PBS/0.1\% Triton-X after each antibody incubation step. Coverslips were mounted on Prolong Glass (Thermo Fisher, Waltham, MA) and cured at room temperature in the dark for two days. Samples were imaged under an Olympus BX2 laser-scanning confocal microscope system equipped with an automated upright BX61 microscope base and PRIOR ProScanII motorized stage. Colocalization of green and red signals was assessed with the ImarisColoc module in Imaris 9.2.0 (b47084) 
software (Bitplane AG, Zurich, Switzerland). Pearson's correlation coefficients (PCC) were calculated for colocalized image volumes across four images within each treatment group to obtain mean PCC values.

\section{Acknowledgement}

We acknowledge the Defense Advanced Research Projects Agency (DARPA) for funding provided under contract number N660011824041. We thank Guillermo Marques, Ph.D., (University of Minnesota - University Imaging Centers) and Thomas Pengo, Ph.D., (University of Minnesota Informatics Institute) for technical advice and consultation.

\section{Supporting Information Available}

The following files are available free of charge.

- RNPHTSsuppinfo.pdf: detailed experimental procedures, modeling workflows, supplemental data from flow cytometry, NMR, $p K_{a}$ titrations, DLS, gel migration, sequencing, confocal imaging, and colocalization analyses.

\section{References}

1. Doudna, J. A.; Charpentier, E. The new frontier of genome engineering with CRISPRCas9. Science 2014, 346 .

2. Doudna, J. A. The promise and challenge of therapeutic genome editing. Nature 2020, $578,229-236$.

3. Zipkin, M. CRISPR's "magnificent moment" in the clinic. Nature Biotechnology 2019,

4. Custom CRISPR therapies could be closer than you think. https://cen.acs.org/ 
business/Custom-CRISPR-therapies-closer-be-closer-than-you-think/97/web/ 2019/10.

5. Sheridan, C. Go-ahead for first in-body CRISPR medicine testing. Nature Biotechnology 2018 ,

6. The first U.S. trials in people put CRISPR to the test in 2019, Science News. https://www.sciencenews.org/article/ crispr-gene-editor-human-clinical-trials-top-science-stories-2019-yir.

7. Lostalé-Seijo, I.; Montenegro, J. Synthetic materials at the forefront of gene delivery. Nature Reviews Chemistry 2018, 2, 258-277.

8. Wilbie, D.; Walther, J.; Mastrobattista, E. Delivery Aspects of CRISPR/Cas for in Vivo Genome Editing. Accounts of Chemical Research 2019, 52, 1555-1564.

9. Li, L.; He, Z. Y.; Wei, X. W.; Gao, G. P.; Wei, Y. Q. Challenges in CRISPR/CAS9 Delivery: Potential Roles of Nonviral Vectors. Human Gene Therapy 2015, 26, 452-462.

10. Xu, C. L.; Ruan, M. Z. C.; Mahajan, V. B.; Tsang, S. H. Viral Delivery Systems for CRISPR. Viruses 2019, 11, 28.

11. Liu, C.; Zhang, L.; Liu, H.; Cheng, K. Delivery strategies of the CRISPR-Cas9 geneediting system for therapeutic applications. J Control Release 2017, 266, 17-26.

12. Wang, H.-X.; Li, M.; Lee, C. M.; Chakraborty, S.; Kim, H.-W.; Bao, G.; Leong, K. W. CRISPR/Cas9-Based Genome Editing for Disease Modeling and Therapy: Challenges and Opportunities for Nonviral Delivery. Chemical Reviews 2017, 117, 9874-9906.

13. Putnam, D. Polymers for gene delivery across length scales. Nature Materials 2006, 5, 439-451.

14. Pack, D. W.; Hoffman, A. S.; Pun, S.; Stayton, P. S. Design and development of polymers for gene delivery. Nature Reviews Drug Discovery 2005, 4, 581-593. 
15. Yin, H.; Kauffman, K. J.; Anderson, D. G. Delivery technologies for genome editing. Nature Reviews Drug Discovery 2017, 16, 387-399.

16. Eoh, J.; Gu, L. Biomaterials as vectors for the delivery of CRISPR-Cas9. Biomater. Sci. 2019, 7, 1240-1261.

17. Sun, H.; Yang, L.; Thompson, M. P.; Schara, S.; Cao, W.; Choi, W.; Hu, Z.; Zang, N.; Tan, W.; Gianneschi, N. C. Recent advances in amphiphilic polymer-oligonucleotide nanomaterials via living/controlled polymerization technologies. 2019, 30, 1889-1904.

18. Vermeulen, L. M.; De Smedt, S. C.; Remaut, K.; Braeckmans, K. The proton sponge hypothesis: Fable or fact? 2018, 129, 184-190.

19. Pichon, C.; Billiet, L.; Midoux, P. Chemical vectors for gene delivery: Uptake and intracellular trafficking. 2010, 21, 640-645.

20. Ong, Z. Y.; Yang, C.; Cheng, W.; Voo, Z. X.; Chin, W.; Hedrick, J. L.; Yang, Y. Y. Biodegradable cationic poly(carbonates): Effect of varying side chain hydrophobicity on key aspects of gene transfection. Acta Biomaterialia 2017, 54, 201-211.

21. Piest, M.; Engbersen, J. F. Effects of charge density and hydrophobicity of poly(amido amine)s for non-viral gene delivery. Journal of Controlled Release 2010, 148, 83-90.

22. Nelson, C. E.; Kintzing, J. R.; Hanna, A.; Shannon, J. M.; Gupta, M. K.; Duvall, C. L. Balancing cationic and hydrophobic content of PEGylated siRNA polyplexes enhances endosome escape, stability, blood circulation time, and bioactivity in vivo. ACS Nano 2013, 7, 8870-8880.

23. Werfel, T. A.; Jackson, M. A.; Kavanaugh, T. E.; Kirkbride, K. C.; Miteva, M.; Giorgio, T. D.; Duvall, C. Combinatorial optimization of PEG architecture and hydrophobic content improves ternary siRNA polyplex stability, pharmacokinetics, and potency in vivo. J. Control. Release 2017, 255, 12-26. 
24. Zhu, D.; Yan, H.; Zhou, Z.; Tang, J.; Liu, X.; Hartmann, R.; Parak, W. J.; Feliu, N.; Shen, Y. Detailed investigation on how the protein corona modulates the physicochemical properties and gene delivery of polyethylenimine (PEI) polyplexes. Biomaterials Science 2018, 6, 1800-1817.

25. Pezzoli, D.; Giupponi, E.; Mantovani, D.; Candiani, G. Size matters for in vitro gene delivery: Investigating the relationships among complexation protocol, transfection medium, size and sedimentation. Scientific Reports 2017, 7, 1-11.

26. Alabi, C. A.; Love, K. T.; Sahay, G.; Yin, H.; Luly, K. M.; Langer, R.; Anderson, D. G. Multiparametric approach for the evaluation of lipid nanoparticles for siRNA delivery. Proc. Natl. Acad. Sci. U. S. A. 2013, 110, 12881-12886.

27. Li, Y.; Yang, T.; Yu, Y.; Shi, N.; Yang, L.; Glass, Z.; Bolinger, J.; Finkel, I. J.; Li, W.; $\mathrm{Xu}, \mathrm{Q}$. Combinatorial library of chalcogen-containing lipidoids for intracellular delivery of genome-editing proteins. Biomaterials 2018, 178, 652-662.

28. Li, Y.; Bolinger, J.; Yu, Y.; Glass, Z.; Shi, N.; Yang, L.; Wang, M.; Xu, Q. Intracellular delivery and biodistribution study of CRISPR/Cas9 ribonucleoprotein loaded bioreducible lipidoid nanoparticles. Biomater. Sci. 2019, 7, 596-606.

29. Billingsley, M. M.; Singh, N.; Ravikumar, P.; Zhang, R.; June, C. H.; Mitchell, M. J. Ionizable Lipid Nanoparticle-Mediated mRNA Delivery for Human CAR T Cell Engineering. Nano Lett. 2020, acs.nanolett.9b04246.

30. Kuhn, J.; Lin, Y.; Krhac Levacic, A.; Al Danaf, N.; Peng, L.; Höhn, M.; Lamb, D. C.; Wagner, E.; Lächelt, U. Delivery of Cas9/sgRNA Ribonucleoprotein Complexes via Hydroxystearyl Oligoamino Amides. Bioconjug. Chem. 2020,

31. Ramishetti, S.; Hazan-Halevy, I.; Palakuri, R.; Chatterjee, S.; Naidu Gonna, S.; Dammes, N.; Freilich, I.; Kolik Shmuel, L.; Danino, D.; Peer, D. A Combinatorial Library of Lipid Nanoparticles for RNA Delivery to Leukocytes. Adv. Mater. 2020, 1906128. 
32. Lynn, D. M.; Anderson, D. G.; Putnam, D.; Langer, R. Accelerated Discovery of Synthetic Transfection Vectors: Parallel Synthesis and Screening of a Degradable Polymer Library. J. Am. Chem. Soc. 2001, 123, 8155-8156.

33. Akin Akinc, David M. Lynn, ; Daniel G. Anderson, Robert Langer*, Akinc, A.; Lynn, D. M.; Anderson, D. G.; Langer, R. Parallel synthesis and biophysical characterization of a degradable polymer library for gene delivery. J. Am. Chem. Soc. 2003, 125, 5316-5323.

34. Anderson, D. G.; Lynn, D. M.; Langer, R. Semi-automated synthesis and screening of a large library of degradable cationic polymers for gene delivery. Angew. Chemie - Int. Ed. 2003, 42, 3153-3158.

35. Green, J. J.; Zugates, G. T.; Tedford, N. C.; Huang, Y. H.; Griffith, L. G.; Lauffenburger, D. A.; Sawicki, J. A.; Langer, R.; Anderson, D. G. Combinatorial modification of degradable polymers enables transfection of human cells comparable to adenovirus. Adv. Mater. 2007, 19, 2836-2842.

36. Anderson, D. G.; Akinc, A.; Hossain, N.; Langer, R. Structure/property studies of polymeric gene delivery using a library of poly( $\beta$-amino esters). Mol. Ther. 2005, 11, $426-434$.

37. Sunshine, J. C.; Akanda, M. I.; Li, D.; Kozielski, K. L.; Green, J. J. Effects of base polymer hydrophobicity and end-group modification on polymeric gene delivery. Biomacromolecules 2011, 12, 3592-3600.

38. Rinkenauer, A. C.; Vollrath, A.; Schallon, A.; Tauhardt, L.; Kempe, K.; Schubert, S.; Fischer, D.; Schubert, U. S. Parallel high-throughput screening of polymer vectors for nonviral gene delivery: Evaluation of structure-property relationships of transfection. ACS Comb. Sci. 2013, 15, 475-482. 
39. Yan, H.; Zhu, D.; Zhou, Z.; Liu, X.; Piao, Y.; Zhang, Z.; Liu, X.; Tang, J.; Shen, Y. Facile synthesis of semi-library of low charge density cationic polyesters from poly(alkylene maleate)s for efficient local gene delivery. Biomaterials 2018, 178, 559-569.

40. Jiang, Y.; Lu, Q.; Wang, Y.; Xu, E.; Ho, A.; Singh, P.; Wang, Y.; Jiang, Z.; Yang, F.; Tietjen, G. T.; Cresswell, P.; Saltzman, W. M. Quantitating Endosomal Escape of a Library of Polymers for mRNA Delivery. 2019,

41. Lee, K.; Conboy, M.; Park, H. M.; Jiang, F.; Kim, H. J.; Dewitt, M. A.; Mackley, V. A.; Chang, K.; Rao, A.; Skinner, C.; Shobha, T.; Mehdipour, M.; Liu, H.; Huang, W. C.; Lan, F.; Bray, N. L.; Li, S.; Corn, J. E.; Kataoka, K.; Doudna, J. A. et al. Nanoparticle delivery of Cas9 ribonucleoprotein and donor DNA in vivo induces homology-directed DNA repair. Nat. Biomed. Eng. 2017, 1, 889-901.

42. Lee, B.; Lee, K.; Panda, S.; Gonzales-Rojas, R.; Chong, A.; Bugay, V.; Park, H. M.; Brenner, R.; Murthy, N.; Lee, H. Y. Nanoparticle delivery of CRISPR into the brain rescues a mouse model of fragile $\mathrm{X}$ syndrome from exaggerated repetitive behaviours. Nat. Biomed. Eng. 2018, 2, 497-507.

43. Fong, T. C.; Bodovitz, S. Polymer nanoparticles : potential for efficient, biodegradable , and cost-effective delivery of gene therapy to multiple tissues. Cell Gene Ther. Insights 2020, 6, 583-590.

44. Freitag, F.; Wagner, E. Optimizing synthetic nucleic acid and protein nanocarriers: The chemical evolution approach. Adv. Drug Deliv. Rev. 2020,

45. Sunshine, J. C.; Akanda, M. I.; Li, D.; Kozielski, K. L.; Green, J. J. Effects of base polymer hydrophobicity and end-group modification on polymeric gene delivery. Biomacromolecules 2011, 12, 3592-3600.

46. Bodnarchuk, M. S.; Doncom, K. E.; Wright, D. B.; Heyes, D. M.; Dini, D.; O’Reilly, R. K. 
Polyelectrolyte pKa from experiment and molecular dynamics simulation. RSC Adv. 2017, 7, 20007-20014.

47. Murmiliuk, A.; Košovan, P.; Janata, M.; Procházka, K.; Uhlík, F.; Štěpánek, M. Local pH and Effective p K of a Polyelectrolyte Chain: Two Names for One Quantity? ACS Macro Lett. 2018, 7, 1243-1247.

48. Nová, L.; Uhlík, F.; Košovan, P. Local pH and effective pKA of weak polyelectrolytesinsights from computer simulations. Phys. Chem. Chem. Phys. 2017, 19, 14376-14387.

49. Certo, M. T.; Ryu, B. Y.; Annis, J. E.; Garibov, M.; Jarjour, J.; Rawlings, D. J.; Scharenberg, A. M. Tracking genome engineering outcome at individual DNA breakpoints. Nature Methods 2011, 8, 671-676.

50. Steyer, B.; Carlson-Stevermer, J.; Angenent-Mari, N.; Khalil, A.; Harkness, T.; Saha, K. High content analysis platform for optimization of lipid mediated CRISPR-Cas9 delivery strategies in human cells. Acta Biomaterialia 2016, 34, 143-158.

51. Chen, G.; Abdeen, A. A.; Wang, Y.; Shahi, P. K.; Robertson, S.; Xie, R.; Suzuki, M.; Pattnaik, B. R.; Saha, K.; Gong, S. A biodegradable nanocapsule delivers a Cas9 ribonucleoprotein complex for in vivo genome editing. 2019, 14, 974-980.

52. Degors, I. M. S.; Wang, C.; Rehman, Z. U.; Zuhorn, I. S. Carriers Break Barriers in Drug Delivery: Endocytosis and Endosomal Escape of Gene Delivery Vectors. Acc. Chem. Res. 2019, 52, 1750-1760.

53. Rejman, J.; Oberle, V.; Zuhorn, I. S.; Hoekstra, D. Size-dependent internalization of particles via the pathways of clathrin-and caveolae-mediated endocytosis. Biochem. J. 2004, 377, 159-169.

54. Khalil, I. A.; Kogure, K.; Akita, H.; Harashima, H. Pharmacol. Rev., 2006, 58, 32.pdf. 2006, $58,32-45$. 
55. Costes, S. V.; Daelemans, D.; Cho, E. H.; Dobbin, Z.; Pavlakis, G.; Lockett, S. Automatic and quantitative measurement of protein-protein colocalization in live cells. Biophys. J. 2004, 86, 3993-4003.

56. Mannhold, R.; Poda, G. I.; Ostermann, C.; Tetko, I. V. Calculation of molecular lipophilicity: State-of-the-art and comparison of $\log \mathrm{P}$ methods on more than 96,000 compounds. Journal of Pharmaceutical Sciences 2009, 98, 861-893.

57. Stubbs, C.; Murray, K. A.; Ishibe, T.; Mathers, R. T.; Gibson, M. I. Combinatorial Biomaterials Discovery Strategy to Identify New Macromolecular Cryoprotectants. ACS Macro Letters 2020, 9, 290-294.

58. Li, Y.; Zhao, T.; Wang, C.; Lin, Z.; Huang, G.; Sumer, B. D.; Gao, J. Molecular basis of cooperativity in pH-triggered supramolecular self-assembly. Nature Communications 2016, भ, 13214.

59. Adolph, E. J.; Nelson, C. E.; Werfel, T. A.; Guo, R.; Davidson, J. M.; Guelcher, S. A.; Duvall, C. L. Enhanced performance of plasmid DNA polyplexes stabilized by a combination of core hydrophobicity and surface PEGylation. J. Mater. Chem. B 2014, 2, 8154-8164.

60. Dual carrier-cargo hydrophobization and charge ratio optimization improve the systemic circulation and safety of zwitterionic nano-polyplexes. Biomaterials 2019, 192, 245-259.

61. Nelson, C. E.; Kintzing, J. R.; Hanna, A.; Shannon, J. M.; Gupta, M. K.; Duvall, C. L. Balancing Cationic and Hydrophobic Content of PEGylated siRNA Polyplexes Enhances Endosome Escape, Stability, Blood Circulation Time, and Bioactivity in Vivo. ACS Nano 2013, \%, 8870-8880.

62. Yan, H.; Zhu, D.; Zhou, Z.; Liu, X. X.; Piao, Y.; Zhang, Z.; Liu, X. X.; Tang, J.; Shen, Y. Facile synthesis of semi-library of low charge density cationic polyesters from 
poly(alkylene maleate)s for efficient local gene delivery. Biomaterials 2018, 178, 559569.

63. Wu, D.; Pusuluri, A.; Vogus, D.; Krishnan, V.; Shields, C. W.; Kim, J.; Razmi, A.; Mitragotri, S. Design principles of drug combinations for chemotherapy. J. Control. Release 2020, 323, 36-46.

64. Tan, Z.; Jiang, Y.; Ganewatta, M. S.; Kumar, R.; Keith, A.; Twaroski, K.; Pengo, T.; Tolar, J.; Lodge, T. P.; Reineke, T. M. Block Polymer Micelles Enable CRISPR/Cas9 Ribonucleoprotein Delivery: Physicochemical Properties Affect Packaging Mechanisms and Gene Editing Efficiency. Macromolecules 2019, 52, 8197-8206.

65. Dosta, P.; Segovia, N.; Cascante, A.; Ramos, V.; Borrós, S. Surface charge tunability as a powerful strategy to control electrostatic interaction for high efficiency silencing, using tailored oligopeptide-modified poly(beta-amino ester)s (PBAEs). Acta Biomater. 2015, 20, 82-93.

66. Dunn, A. W.; Kalinichenko, V. V.; Shi, D. Highly Efficient In Vivo Targeting of the Pulmonary Endothelium Using Novel Modifications of Polyethylenimine: An Importance of Charge. Adv. Healthc. Mater. 2018, 7, 1800876.

67. Fröhlich, E. The role of surface charge in cellular uptake and cytotoxicity of medical nanoparticles. Int. J. Nanomedicine 2012, 7, 5577-5591.

68. Staedtler, A. M.; Hellmund, M.; Sheikhi Mehrabadi, F.; Thota, B. N.; Zollner, T. M.; Koch, M.; Haag, R.; Schmidt, N. Optimized effective charge density and size of polyglycerol amines leads to strong knockdown efficacy in vivo. J. Mater. Chem. B 2015, 3, 8993-9000.

69. Ghosh, P. S.; Kim, C. K.; Han, G.; Forbes, N. S.; Rotello, V. M. Efficient gene delivery vectors by tuning the surface charge density of amino acid-functionalized gold nanoparticles. ACS Nano 2008, 2, 2213-2218. 


\section{Graphical TOC Entry}

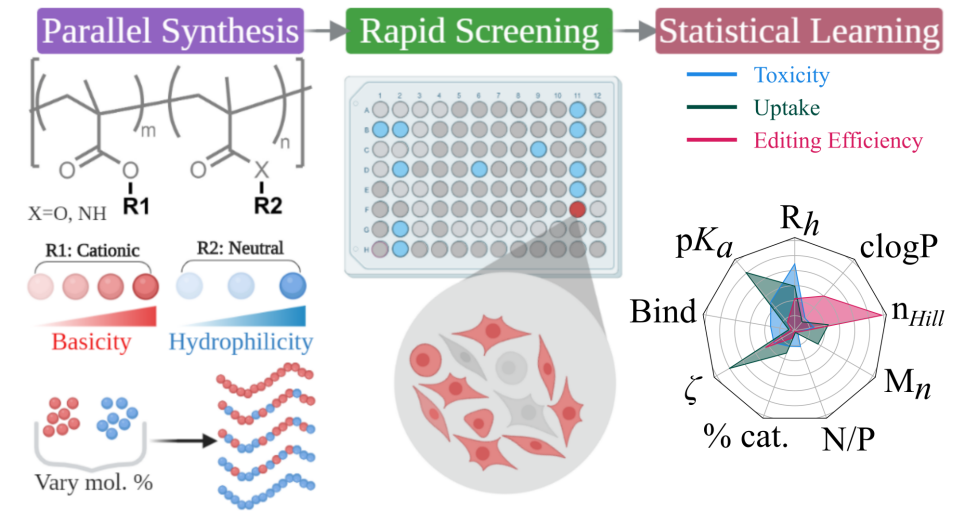

